\title{
Momentum distribution and contacts of one-dimensional spinless Fermi gases with an attractive $p$-wave interaction
}

\author{
Xiangguo Yin, ${ }^{1,2,{ }^{*}}$ Xi-Wen Guan, ${ }^{3,4}$ Yunbo Zhang, ${ }^{1}$ Haibin $\mathrm{Su},{ }^{5}$ and Shizhong Zhang ${ }^{2}$ \\ ${ }^{1}$ Institute of Theoretical Physics, Shanxi University, Taiyuan 030006, China \\ ${ }^{2}$ Department of Physics and Center of Theoretical and Computational Physics, The University of Hong Kong, Hong Kong, China \\ ${ }^{3}$ State Key Laboratory of Magnetic Resonance and Atomic and Molecular Physics, Wuhan Institute of Physics and Mathematics, \\ Chinese Academy of Sciences, Wuhan 430071, People's Republic of China \\ ${ }^{4}$ Department of Theoretical Physics, Research School of Physics and Engineering, Australian National University, \\ Canberra ACT 0200, Australia \\ ${ }^{5}$ Department of Chemistry, The Hong Kong University of Science and Technology, Hong Kong, China
}

(Received 13 March 2018; published 7 August 2018)

\begin{abstract}
We present a rigorous study of momentum distribution and $p$-wave contacts of one-dimensional spinless Fermi gases with an attractive $p$-wave interaction. Using the Bethe ansatz wave function, we calculate analytically the high-momentum tail and show that the leading $\left(\sim 1 / p^{2}\right)$ and sub-leading terms $\left(\sim 1 / p^{4}\right)$ are determined by two contacts $C_{2}$ and $C_{4}$, which are related to the short-distance behavior of the two-body density matrix and its derivatives. As one increases the one-dimensional scattering length, the contact $C_{2}$ increases monotonically from zero while $C_{4}$ exhibits a peak at finite scattering length. In addition, we obtain analytic expressions for $p$-wave contacts at finite temperature from the thermodynamic Bethe ansatz equations for both weak and strong attractive interactions.
\end{abstract}

DOI: 10.1103/PhysRevA.98.023605

\section{INTRODUCTION}

In the past few decades, experimental advances have made it possible to engineer with high controllability one-dimensional systems in ultracold atoms. Furthermore, the interactions between atoms can be tuned by a variety of experimental techniques, thus offering a promising opportunity to realize one-dimensional (1D) models of interacting spins, bosons, and fermions. It is well known that ultracold atomic gases in 1D display a rich variety of few-body to many-body physics [1-4]. In contrast to the study of quantum many-body systems in higher dimensions, many 1D systems can be treated in exact manners, such as the Bethe ansatz approach [5,6], Bose-Fermi mapping [7], and quantum field theory [8].

Exactly solvable models provide an important benchmark understanding of quantum many-body phenomena, including quantum correlations, quantum criticality, and quantum liquids [9-12]. In this regard, the prototypical exactly solvable model of the Lieb-Liniger Bose gas provides a deep understanding of quantum statistics, thermodynamics, and quantum critical phenomena [13,14]; see review [15]. The Bethe ansatz solution of this model is not only widely used to perform analytical calculations of important physical quantities which shed light on universal behavior of many-body systems, but also presents a test ground to explore equilibrium and nonequilibrium physics in experiment, for example, Tonks-Girardeau gases [3,4], super Tonks-Girardeau gases [16], quantum liquids [17], and thermalization of 1D ensembles of cold atoms $[18,19]$.

\footnotetext{
*yinxiangguo@sxu.edu.cn
}

By using optical lattices, low-dimensional quantum gases with rich internal degrees of freedom can be realized. These advances in experiments stimulate growing theoretical interests in 1D quantum spinor gases with high spin symmetries and high partial wave interactions. In the strong-coupling regime, these systems display very different patterns of spin-charge separations depending on the statistics of constituent particles [20-27].

A universal theme in the study of dilute atomic gas is the correspondence between two-body and many-body correlations at short distance [28-33]. This correspondence manifests itself in the relation between various physical quantities for which the so-called contact plays a central role. These relations include the adiabatic sweep relation, the high-momentum tails, the derivative of the free energy with respect to scattering length, the virial theorem, pressure relation, and so on. A nice feature of these relations is that they apply to both bosons and fermions in any dimensions, in both high and low temperatures, irrespective of the states of the system.

Very recently, universal relations are found in systems of ultracold atoms with $p$-wave interactions [34-41]. For spinless Fermi gas, there is no $s$-wave interaction and $p$-wave interaction dominates. At low energies, the phase shift $\delta_{p}(k)$ of $p$-wave interaction in three-dimensional (3D) space can be expressed as

$$
k^{3} \cot \delta_{p}(k)=-\frac{1}{v}-\frac{k^{2}}{R}+O\left(k^{4}\right),
$$

where $k$ is the relative momentum of colliding atoms and $v$ is the $p$-wave scattering volume and $R$ the effective range. An important difference from the $s$-wave contact theory is that in the $p$-wave case, the effective range $R$ is also a relevant 
parameter that needs to be taken into account on the same footing as $v$ [35].

The $p$-wave contact theory has also been extended to 1D. In Ref. [38], the operator production method is employed to derive the universal relations using a two-channel model, including both effects of $v$ and $R$. As in the 3D case, a set of universal relations is established. However, no explicit calculation is given for the values of contacts except for a two-body calculation. On the other hand, Sekino et al. [36] used Bose-Fermi mapping to establish relations of correlations functions in 1D Bose and Fermi gas models, and consequently, the relation between contacts in Bose and Fermi models. In their calculation, they have ignored the effects of effective range (which is not as important in $1 \mathrm{D}$ as in 3D).

In this paper, we aim to obtain explicitly, using Bethe ansatz (BA), the expressions for contacts for a 1D $p$-wave Fermi gas, including the effects of effective range. In addition, we aim to verify, through explicit computation, the various universal relations derived on general grounds. In particular, we show that the leading and subleading terms $\left(\sim 1 / p^{2}\right.$ and $\left.\sim 1 / p^{4}\right)$ of the high-momentum tail give rise to two contacts $C_{2}$ and $C_{4}$, respectively, which are determined by the two-body density matrix and its derivative in the short range.

The paper is organized as follows. In Sec. II we discuss the interaction boundary condition and the BA solution of of the $1 \mathrm{D}$ spinless Fermi gases with a $p$-wave interaction. In Sec. III, we present our main results of the $p$-wave contacts, the high-momentum asymptotic of the momentum distribution. We find that the coefficients of the universal leading $1 / p^{2}$ and subleading $1 / p^{4}$ tails are related to the two-body correlation function and its derivative, respectively. In Sec. IV we calculate the two-body correlation function and contacts in the weakcoupling regime, and test the relationship between correlation function and energy derivatives. We also show the consistence between the statistical approach and the thermodynamic Bethe ansatz (TBA) method. In addition, we discuss the contacts in the strong attractive regime. We conclude the result of our work in Sec. V.

\section{MODEL}

We consider a 1D system composed of spinless Fermi gas with a $p$-wave interaction, which implies the following boundary condition for wave function $\psi(\vec{z})[42,43]$ :

$$
\lim _{z=z_{j}-z_{i} \rightarrow 0^{+}}\left(\frac{1}{a_{p}^{1 \mathrm{D}}}+\partial_{z}-R^{1 \mathrm{D}} \partial_{z}^{2}\right) \psi(\vec{z})=0,
$$

where $\vec{z}=z_{1}, \ldots, z_{N}$ are coordinates of $N$ fermions. Under a strong two-dimensional harmonic confinement, only the lowest transverse mode is occupied, and the 1D scattering length $a_{p}^{1 \mathrm{D}}$ and 1D effective range $R^{1 \mathrm{D}}$ are related to the 3D scattering volume $v$ and 3D effective range $R$ through [42]

$$
a_{p}^{1 \mathrm{D}}=3 a_{\perp}\left[\frac{a_{\perp}^{3}}{v}-3 \sqrt{2} \zeta(-1 / 2)\right]^{-1}, \quad R^{1 \mathrm{D}}=\frac{a_{\perp}^{2}}{3 R},
$$

respectively. Here $3 \sqrt{2} \zeta(-1 / 2) \approx-0.88$. The relations in (3) require that the momentum of scattering fermions satisfy $k a_{\perp} \ll 1$. As usual, $a_{\perp}=\sqrt{\hbar / m \omega_{\perp}}$ is the transverse oscillator length, $m$ is the atomic mass, and $\omega_{\perp}$ is the transverse trapping frequency. The derivation of 1D scattering length and 1D effective range is related to solution of Lippmann-Schwinger equation in cylindrical coordinates [42].

By using the above boundary condition (2) and the asymptotic Bethe ansatz, the eigenvalues and eigenfunctions of the uniform system have been exactly obtained $[43,44]$. The wave function in the domain $\left\{0 \leqslant z_{1} \leqslant \cdots \leqslant z_{N} \leqslant L\right\}$ is given in terms of the superpositions of $N$ ! plane waves,

$$
\psi(\vec{z})=\sum_{P} a(P) \exp \left(i \sum_{l=1}^{N} \lambda_{P_{l}} z_{l}\right),
$$

where $\lambda_{1}, \ldots, \lambda_{N}$ are quasimomenta. In the above equation, the summation accounts for all permutations $P$ s of the $N$ numbers $1,2, \ldots, N$, and $a(P)$ stands for the coefficients depending on the quasimomenta, $p$-wave interaction parameters, i.e., the scattering length $a_{p}^{1 \mathrm{D}}$ and the effective range $R^{1 \mathrm{D}}$. The wave function in other domains can be obtained by symmetry considerations. The energy of system is given by $E=\hbar^{2} /(2 m) \sum_{i=1}^{N} \lambda_{i}^{2}$. In the following calculation, we take the units $\hbar=2 m=1$.

Using the interaction boundary condition (2) and the wave function with periodic boundary conditions (4), we obtain the amplitudes

$$
a(P)=(-1)^{P} \prod_{i<j=1}^{N}\left[S\left(\lambda_{P_{i}}-\lambda_{P_{j}}\right)\right]^{1 / 2},
$$

where

$$
S(x)=\frac{\xi_{p} x^{2}-1 /\left|l_{p}\right|+i x}{\xi_{p} x^{2}-1 /\left|l_{p}\right|-i x} .
$$

Here we denote the scattering length $l_{p}=a_{p}^{1 \mathrm{D}} / 2$ and the effective range $\xi_{p}=R^{1 \mathrm{D}} / 2$. Note that $(-1)^{P}=+1(-1)$ for an even (odd) permutation. It follows that the BA equations read [43]

$$
\exp \left(i \lambda_{i} L\right)=\prod_{j=1}^{N} S\left(\lambda_{i}-\lambda_{j}\right), \quad i=1,2, \ldots, N,
$$

which determines the value of quasimomenta $\lambda_{1}, \ldots, \lambda_{N}$. The BA equations provide exact ground state and excitations of the $1 \mathrm{D}$ spinless fermions with $p$-wave interactions. For $l_{p}=0$, the BA equations (7) naturally reduce to the quasimomenta of free fermions, for which the wave function $\psi(\vec{z})$ is given by Slater determinant, whereas at $p$-wave resonance, i.e., $l_{p}=$ $\infty$, the BA equations (7) reduce to those of the Lieb-Liniger Bose gas [13] with coupling constant $c_{B}=1 / \xi_{p}$. At the $p$ wave resonance, a large value of $\xi_{p}$ drives the $p$-wave spinless Fermi gas into the regime of the weakly interacting bosons. This gives a very interesting physical regime and is likely to be reachable in experiment. In contrast, for $\xi_{p}=0$, the BA equations (7) reduce to the ones for the 1D Lieb-Liniger Bose gas with interacting strength $c=1 /\left|l_{p}\right|$, reversing the BoseFermi mapping. The instability of this model was discussed in [45]. In this paper, we only focus the case of $a_{p}^{1 \mathrm{D}}<0$ and $R^{1 \mathrm{D}}>0$, for which the solutions of quasimomenta are real. The general solutions to the BA equations (7) are much more complicated. As usual, by taking logarithm of both sides of the 
equations (7), the roots are determined by

$$
\lambda_{i} L=2 \pi J_{i}-\sum_{j=1}^{N} \theta\left(\lambda_{i}-\lambda_{j}\right),
$$

where the phase shift is given by

$$
\theta(\lambda)=2 \arg \left(i \lambda-\xi_{p} \lambda^{2}+1 /\left|l_{p}\right|\right) .
$$

In the above equations, the quantum numbers $J_{i}$ take an integer (half an integer) for odd (even) particle number $N$. The thermodynamics of this model is presented in [46].

\section{MOMENTUM DISTRIBUTION}

We are interested in the asymptotic behavior of the momentum distribution, which determines the $p$-wave contacts. The dominant contribution in the high-momentum tail involves the singular behavior of the wave function in the vicinity of the interaction point, i.e., $z_{i j}=z_{i}-z_{j} \rightarrow 0$. In order to evaluate it, here we generalize the method for calculating the high-momentum distribution $[36,47]$ and the method for calculating the multiparticle local correlation functions [48]. To this end, three major steps are needed: (a) a short-range expansion of the wave function in the vicinity of the interaction point; (b) the asymptotics of Fourier integral of the wave function; and (c) two-body correlation functions. We discuss the major step for calculating the correlation function in the next section.

A short-range expansion of the wave function. Without losing generality, we consider the interaction point of the first and the $i$ th particles. The wave function in the domain $\left\{0 \leqslant z_{2}<z_{3}<\cdots<z_{N} \leqslant L\right\}$ can be expanded in terms of $z_{i 1} \equiv z_{1}-z_{j}$ :

$$
\begin{aligned}
\psi\left(z_{1}, \ldots, z_{N}\right)= & \psi_{0}\left(Z_{i}\right) \operatorname{sgn}\left(z_{i 1}\right)+\psi_{1}\left(Z_{i}\right) z_{i 1} \\
& +\psi_{2}\left(Z_{i}\right) \operatorname{sgn}\left(z_{i 1}\right) z_{i 1}^{2}+O\left(z_{i 1}^{3}\right)
\end{aligned}
$$

with

$$
\begin{gathered}
\psi_{0}\left(Z_{i}\right)=(-1)^{i} \sum_{P} a(P) J\left(P, Z_{i}\right), \\
\psi_{1}\left(Z_{i}\right)=(-1)^{i} \sum_{P} a(P) J\left(P, Z_{i}\right)\left(\frac{i}{2}\right)\left(k_{P_{i}}-k_{P_{i-1}}\right), \\
\psi_{2}\left(Z_{i}\right)=(-1)^{i} \sum_{P} a(P) J\left(P, Z_{i}\right)\left(-\frac{1}{8}\right)\left(k_{P_{i}}-k_{P_{i-1}}\right)^{2} .
\end{gathered}
$$

Here $\quad J\left(P, Z_{i}\right)=\exp \left\{i\left[\left(k_{P_{i-1}}+k_{P_{i}}\right) Z_{i 1}+\sum_{j=1}^{i-2} k_{P_{j}} z_{j+1}+\right.\right.$ $\left.\left.\sum_{j=i+1}^{N} k_{P_{j}} z_{j}\right]\right\}, \quad Z_{i 1}=\left(z_{i}+z_{1}\right) / 2, \quad$ and $\quad z_{i 1}=z_{i}-z_{1}$ are the center-of-mass and relative coordinates of the (1i) pair of particles, respectively. We will denote by $Z_{i}=\left\{Z_{i 1}, z_{2}, \ldots, z_{i-1}, z_{i+1}, \ldots, z_{N}\right\}$ the center-of-mass coordinate of the first and $i$ th particles and the coordinates of all the rest of the particles. In the above equations the sign function is defined as $\operatorname{sgn}(z)=-1$ for $z<0, \operatorname{sgn}(z)=0$ for $z=0$, and $\operatorname{sgn}(z)=+1$ for $z>0$. Due to the Pauli exclusion principle, the wave function is zero at the interaction point, and it is not continuous due to the $p$-wave interaction for the 1D spinless Fermi gas with an attractive $p$-wave interaction.
The wave function in the other domains has a similar result by taking into account its antisymmetry.

The asymptotics of the Fourier integral of the wave function. In general, for periodic functions $\operatorname{sgn}\left(z_{0}-z\right) F(z)$, $\left(z_{0}-z\right) F(z)$, and $\operatorname{sgn}\left(z_{0}-z\right)\left(z_{0}-z\right)^{2} F(z)$, which are defined on the interval $[0, L]$, where $F(z)$ is a regular function, we can directly calculate their Fourier transforms through integration by parts. Up to the order of $1 / p^{3}$, we obtain asymptotics of the Fourier transforms of these functions,

$$
\begin{gathered}
\int_{0}^{L} d z e^{-i p z} \operatorname{sgn}\left(z_{0}-z\right) F(z) \\
=\left[\left(\frac{2 i}{p}+\frac{2}{p^{2}} \partial_{z}-\frac{2 i}{p^{3}} \partial_{z}^{2}\right) F(z)\right]_{z=z_{0}} e^{-i p z_{0}}+O\left(\frac{1}{p^{4}}\right) \\
\int_{0}^{L} d z e^{-i p z}\left(z_{0}-z\right) F(z)=0 \\
\int_{0}^{L} d z e^{-i p z} \operatorname{sgn}\left(z_{0}-z\right)\left(z_{0}-z\right)^{2} F(z) \\
=-\frac{4 i}{p^{3}} e^{-i p z_{0}} F\left(z_{0}\right)+O\left(\frac{1}{p^{4}}\right)
\end{gathered}
$$

where $p=2 \pi s / L$ and $s$ is an integer. For multiple singular points, the asymptotic of the Fourier transform of the wave function is given by the sum of the corresponding terms (14), (15), and (16). Using (10), and (14), (15), (16), the momentum representation of the wave function with respect to the first particle reads

$$
\begin{aligned}
& \psi\left(p, z_{2}, \ldots, z_{N}\right) \\
& =\frac{1}{\sqrt{2 \pi}} \int_{0}^{L} d z_{1} e^{-i p z_{1}} \psi(\vec{z}) \\
& \approx \\
& \quad \frac{1}{\sqrt{2 \pi}} \int_{0}^{L} d z_{1} e^{-i p z_{1}} \sum_{i=2}^{N}\left[\psi_{0}\left(Z_{i}\right) \operatorname{sgn}\left(z_{i 1}\right)\right. \\
& \left.\quad+\psi_{1}\left(Z_{i}\right) z_{i 1}+\psi_{2}\left(Z_{i}\right) \operatorname{sgn}\left(z_{i 1}\right) z_{i 1}^{2}+\cdots\right] \\
& \approx \sum_{i=2}^{N} \frac{1}{\sqrt{2 \pi}} e^{-i p z_{i}}\left[\left(\frac{2 i}{p}+\frac{2}{p^{2}} \partial_{z_{1}}-\frac{2 i}{p^{3}} \partial_{z_{1}}^{2}\right) \psi_{0}\left(Z_{i}\right)\right. \\
& \left.\quad-\frac{4 i}{p^{3}} \psi_{2}\left(Z_{i}\right)\right]_{z_{1}=z_{i}-\varepsilon},
\end{aligned}
$$

which can be used to compute the momentum distribution in an analytical way. Here $\varepsilon$ is positive infinitesimal, and the last two expressions are the approximate results for $|p| \rightarrow \infty$.

The momentum distribution is obtained by a multiple integral of $\left|\psi\left(p, z_{2}, \ldots, z_{N}\right)\right|^{2}$ with respect to $z_{2}, z_{3}, \ldots, z_{N}$ :

$$
\begin{aligned}
w(p) & =N \int_{0}^{L} d z_{2} \cdots \int_{0}^{L} d z_{N}\left|\psi\left(p, z_{2}, \ldots, z_{N}\right)\right|^{2} \\
& \stackrel{|p| \rightarrow \infty}{\approx} \frac{C_{2}}{p^{2}}+\frac{C_{3}}{p^{3}}+\frac{C_{4}}{p^{4}},
\end{aligned}
$$


where the coefficients $C$ 's are regarded as the contacts [28], namely,

$$
\begin{gathered}
C_{2}=D \int_{0}^{L} d z_{2} \cdots \int_{0}^{L} d z_{N}\left|\psi_{0}\left(Z_{2}\right)\right|_{z_{1}=z_{2}-\varepsilon}^{2} \\
C_{3}=\left.2 D \int_{0}^{L} d z_{2} \cdots \int_{0}^{L} d z_{N} \operatorname{Im}\left[\psi_{0}^{*}\left(Z_{2}\right) \partial_{z_{1}} \psi_{0}\left(Z_{2}\right)\right]\right|_{z_{1}=z_{2}-\varepsilon} \\
C_{4}=D \int_{0}^{L} d z_{2} \cdots \int_{0}^{L} d z_{N}\left[\left|\partial_{z_{1}} \psi_{0}\left(Z_{2}\right)\right|^{2}\right. \\
\left.-2 \operatorname{Re}\left(\psi_{0}^{*}\left(Z_{2}\right)\left[\partial_{z_{1}}^{2} \psi_{0}\left(Z_{2}\right)+2 \psi_{2}\left(Z_{2}\right)\right]\right)\right]_{z_{1}=z_{2}-\varepsilon}
\end{gathered}
$$

Here $D=2 N(N-1) / \pi$, and $\operatorname{Re}(x), \operatorname{Im}(x)$ denote the real part and imaginary part of the function $x$, respectively. In deriving formula (18), we have retained the direct terms but dropped the cross terms with respect to the summation over $i$ in Eq. (17). In the high-momentum limit, the integrand functions include oscillatory exponential functions in the cross terms that approach to zero upon integration and can be dropped.

In order to evaluate the high-momentum tail, we define the two-body correlation function

$$
\begin{aligned}
& \frac{g_{2}\left(y_{1}, y_{2} ; z_{1}, z_{2}\right)}{N(N-1)} \\
& =\frac{\int_{0}^{L} d z_{3} \cdots \int_{0}^{L} d z_{N} \psi^{*}\left(y_{1}, y_{2}, z_{3}, \ldots, z_{N}\right) \psi(\vec{z})}{\int_{0}^{L} d z_{1} \cdots \int_{0}^{L} d z_{N}|\psi(\vec{z})|^{2}} .
\end{aligned}
$$

Due to the antisymmetry of the wave function, the local twobody correlation function $g_{2}(0,0 ; 0,0)$ vanishes. However, the $p$-wave boundary conditions [Eq. (2)] impose a discontinuity of the wave function in the vicinity of the interaction point. As a result, the quasilocal two-body correlation function $\lim _{\varepsilon \rightarrow 0} g_{2}(0, \varepsilon ; 0, \varepsilon)$ reveals the nature of the $p$-wave contacts. It gives the probability of finding two fermions staying in a short distance $\varepsilon$. It appears to be nonzero for finite interaction strength. For the homogeneous system, the two-body correlation function is translational invariant, and therefore $C_{2}$ is proportional to the quasilocal two-body correlation function. Whereas the other contacts $C_{3}$ and $C_{4}=C_{4}^{c}+C_{4}^{r}$ can be expressed in terms of derivatives of the two-body correlation function, namely,

$$
\begin{gathered}
C_{2}=\frac{2 L}{\pi} g_{2}(0, \varepsilon ; 0, \varepsilon), \\
C_{3}=\left.\frac{2 L}{\pi}\left[\operatorname{Im} \partial_{z_{2}}\right] g_{2}\left(y_{2}, y_{2}+\varepsilon ; z_{2}, z_{2}+\varepsilon\right)\right|_{y_{2}=z_{2}=0}, \\
C_{4}^{c}=\frac{L}{2 \pi}\left[\partial_{y_{2}} \partial_{z_{2}}-2 \operatorname{Re} \partial_{z_{2}}^{2}\right] \\
\times\left. g_{2}\left(y_{2}, y_{2}+\varepsilon ; z_{2}, z_{2}+\varepsilon\right)\right|_{y_{2}=z_{2}=0}, \\
C_{4}^{r}=-\frac{L}{\pi}\left[\operatorname{Re}\left(\partial_{z_{2}}-\partial_{z_{1}}\right)^{2}\right] \\
\times\left. g_{2}\left(y_{2}, y_{2}+\varepsilon ; z_{1}, z_{2}+\varepsilon\right)\right|_{y_{2}=z_{2}=z_{1}=0} .
\end{gathered}
$$

Here $C_{4}^{c}$ is related to the derivatives of correlation function with respect to its two coordinates together, which is related to the center-of-mass movements of the pairs, while $C_{4}^{r}$ is related to the difference of the derivatives of correlation function with respect to $z_{1}$ and $z_{2}$, indicating a contribution from the relative
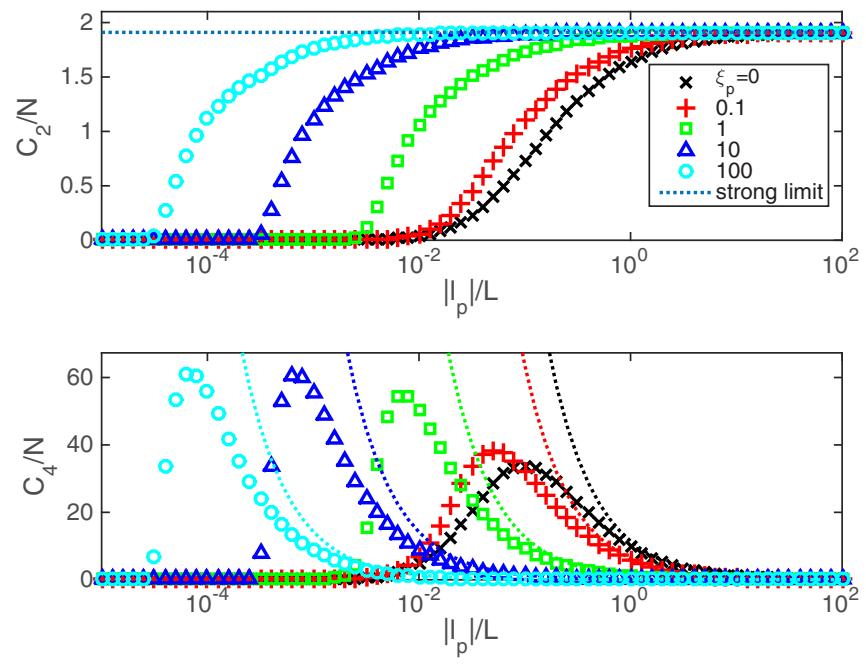

FIG. 1. The contacts of $C_{2}$ and $C_{4}$ at the ground state of the model with particle number $N=4$. The symbols denote the numerical curves determined from Eqs. (23), (25), and (26), whereas the dashed lines are the strong-coupling result from (65).

motion of the pairs. For the ground state or thermodynamic equilibrium state without breaking inversion symmetry, the momentum distribution is symmetric about $p=0$, and it is obvious that $C_{3}=0$, which is indeed the case after explicit calculations. Consequently, the momentum distribution at a high-momentum tail has two terms,

$$
w(p) \stackrel{|p| \rightarrow \infty}{=} \frac{C_{2}}{p^{2}}+\frac{C_{4}}{p^{4}}
$$

which determines the two $p$-wave contacts.

In Fig. 1 we show the numerical result of $C_{2}$ and $C_{4}$ for the ground state of the model with four particles. When the scattering length $l_{p}=0$, the model behaves as the ideal Fermi gas with the zero values of contacts $C_{2}=C_{4}=0$. When the scattering length $\left|l_{p}\right|$ increases, the fermions prefer to stay together due to the attractive interaction that leads to an increase of the contact $C_{2}$. Here we observe that $C_{2}$ increases more quickly for a larger value of the range $\xi_{p}$. When $l_{p} \rightarrow \infty$, $C_{2}$ saturates to the limit of strongly interacting case, which are the same for various $\xi_{p}$ 's. On the other hand, with the increase of $\left|l_{p}\right|, C_{4}$ first grows to a maximal value and then decreases rapidly to zero. The peak positions of $C_{4}$ move to small values of $\left|l_{p}\right|$ for increasing values of the effective range $\xi_{p}$.

\section{CORRELATION FUNCTION}

In this section, we present a straightforward calculation of the contact $C_{2}$ and $C_{4}$ for weak interaction regime, i.e., $\left|l_{p}\right| \ll 1 / 2 \pi n$ and $\xi_{p}<1 / 2 \pi n$, where the particle number density $n=N / L$. Following the method used for calculating high-order local and nonlocal correlation functions of 1D strong interaction Bose gas [48], we may directly calculate the correlation functions of the 1D $p$-wave Fermi gases up to the second order of $\left|l_{p}\right|$. 


\section{A. Weak interaction}

For weak interaction, i.e., $\left|l_{p}\right| \ll 1 / 2 \pi n$ and $\xi_{p}<1 / 2 \pi n$, the coefficients $a(P)$ in the wave function can be expanded up to the second order of $\left|l_{p}\right|$ with the following form:

$$
\begin{aligned}
a(P) \approx & (-1)^{P} \prod_{i<j=1}^{N}\left(1-\left|l_{p}\right| i \lambda_{P_{i} P_{j}}\right. \\
& \left.-\frac{1}{2} l_{p}^{2} \lambda_{P_{i} P_{j}}^{2}\left[1+2 i \xi_{p} \lambda_{P_{i} P_{j}}\right]\right),
\end{aligned}
$$

with $\lambda_{m l}=\lambda_{m}-\lambda_{l}$ and as a result the wave function in the domain $\left\{0 \leqslant z_{1} \leqslant \cdots \leqslant z_{N} \leqslant L\right\}$ for $z_{2}=z_{1}+\varepsilon$ has the new form

$$
\psi(\vec{z})=\psi^{(1)}(\vec{z})\left|l_{p}\right|+\psi^{(2)}(\vec{z}) l_{p}^{2},
$$

in which the wave function to the first order and the second order of $\left|l_{p}\right|$ are given by

$$
\begin{gathered}
\psi^{(1)}(\vec{z})=\left(\partial_{z_{2}}-\partial_{z_{1}}\right) \psi^{(0)}(\vec{z}), \\
\psi^{(2)}(\vec{z})=-\left[\xi_{p}\left(\partial_{z_{2}}-\partial_{z_{1}}\right)^{2}+(N-2)\left(\partial_{z_{2}}+\partial_{z_{1}}\right)\right. \\
\left.+\sum_{i=3}^{N}(N+1-2 i) \partial_{z_{i}}\right] \psi^{(1)}(\vec{z}),
\end{gathered}
$$

respectively. Here the zeroth-order wave function $\psi^{(0)}$ has the form of a Slater determinant $\psi^{(0)}(\vec{z})=\sum_{P}(-1)^{P} e^{i \sum_{j=1}^{N} \lambda_{P_{j}} z_{j}}$. Without loss of generality we shall assume that $\lambda_{1}<\lambda_{2}<$ $\cdots<\lambda_{N}$. Using the wave function (29), we show that up to the order of $\left|l_{p}\right|^{3}$, the numerator of the two-body correlation function (22) for a positive infinitesimal $\varepsilon$ reads (for details, see calculation in Appendix A)

$$
\begin{aligned}
g_{2}\left(y_{1}, y_{2} ; z_{1}, z_{2}\right)= & \left\{1-\left|l_{p}\right| \xi_{p}\left[\left(\partial_{y_{2}}-\partial_{y_{1}}\right)^{2}+\left(\partial_{z_{2}}-\partial_{z_{1}}\right)^{2}\right]\right\} \\
& \times g_{2}^{(2)}\left(y_{1}, y_{2} ; z_{1}, z_{2}\right) .
\end{aligned}
$$

Here the second-order correlation function is defined as the following derivatives:

$$
\begin{aligned}
g_{2}^{(2)}\left(y_{1}, y_{2} ; z_{1}, z_{2}\right)= & l_{p}^{2}\left(\partial_{y_{2}}-\partial_{y_{1}}\right)\left(\partial_{z_{2}}-\partial_{z_{1}}\right) \\
& \times g_{2}^{(0)}\left(y_{1}, y_{2} ; z_{1}, z_{2}\right) .
\end{aligned}
$$

In the above calculation, we have omitted terms of higher order in $\left|l_{p}\right| \xi_{p}$. We define the zeroth-order correlation function

$$
\begin{aligned}
g_{2}^{(0)}\left(y_{1}, y_{2} ; z_{1}, z_{2}\right)= & \int_{0}^{L} d z_{3} \cdots \int_{0}^{L} d z_{N} \psi^{(0)}(\vec{z}) \\
& \times\left[\psi^{(0)}\left(y_{1}, y_{2}, z_{3}, \ldots, z_{N}\right)\right]^{*} .
\end{aligned}
$$

This zeroth-order correlation function goes to zero when we take the short-distance limit $\varepsilon \rightarrow 0$ [due to the vanishing of the zeroth-order wave function $\left.\psi^{(0)}(\vec{z})\right]$. However, its derivatives give nonzero results when taking the limit $\varepsilon \rightarrow 0$.

From BA equations (7) with the weak interaction, we can obtain the solution $\lambda_{i}=\lambda_{i}^{F} \alpha$ for total momentum $\sum_{i} \lambda_{i}=0$, where $\lambda_{i}^{F}=2 \pi J_{i} / L, \alpha=1-2\left|l_{p}\right| n$, and the $J_{i}$ are (half-)integers satisfying $J_{1}<J_{2}<\cdots<J_{N}$. Under the scaling $z_{i}=z_{i}^{F} / \alpha$, the zeroth-order wave function $\psi^{(0)}(\vec{z})$ is identical to the form for ideal Fermi gas $\psi^{F}\left(\overrightarrow{z^{F}}\right)=$ $\sum_{P}(-1)^{P} e^{i \sum_{j=1}^{N} \lambda_{P_{j}}^{F} z_{j}^{F}}$ with $\overrightarrow{z^{F}}=z_{1}^{F}, \ldots, z_{N}^{F}$. We also find the normalization condition [48]

$$
\begin{aligned}
& \int_{0}^{L} d z_{1} \cdots \int_{0}^{L} d z_{N}|\psi(\vec{z})|^{2} \\
& \quad=\alpha^{1-N} \int_{0}^{L} d z_{1}^{F} \cdots \int_{0}^{L} d z_{N}^{F}\left|\psi^{F}\left(\overrightarrow{z^{F}}\right)\right|^{2} .
\end{aligned}
$$

Then the zeroth-order correlation function with a normalization factor has the following form:

$$
\begin{aligned}
& \frac{g_{2}^{(0)}\left(y_{1}, y_{2} ; z_{1}, z_{2}\right)}{\alpha N(N-1)} \\
& =\frac{\int_{0}^{L} d z_{3}^{F} \cdots \int_{0}^{L} d z_{N}^{F}\left[\psi^{F}\left(y_{1}^{F}, y_{2}^{F}, z_{3}^{F}, \ldots, z_{N}^{F}\right)\right]^{*} \psi^{F}\left(\overrightarrow{z^{F}}\right)}{\int_{0}^{L} d z_{1}^{F} \cdots \int_{0}^{L} d z_{N}^{F}\left|\psi^{F}\left(\overrightarrow{z^{F}}\right)\right|^{2}} .
\end{aligned}
$$

Since $\psi^{F}\left(\overrightarrow{z^{F}}\right)$ is a Slater determinant, one can use Wick's theorem [48],

$$
\begin{aligned}
g_{2}^{(0)}\left(y_{1}, y_{2} ; z_{1}, z_{2}\right)= & \alpha\left[G\left(y_{1}^{F}, z_{1}^{F}\right) G\left(y_{2}^{F}, z_{2}^{F}\right)\right. \\
& \left.-G\left(y_{1}^{F}, z_{2}^{F}\right) G\left(y_{2}^{F}, z_{1}^{F}\right)\right],
\end{aligned}
$$

where the single-particle reduced density matrix of ideal fermions is given by $G(y, z)=\frac{1}{L} \sum_{i=1}^{N} \exp \left[-i \lambda_{i}^{F}(y-z)\right]$. Substituting the above formula into expression (33), then substituting (33) into Eq. (32) and after a lengthy calculation, we obtain the two-body correlation function (for details, see calculation in Appendix A),

$$
\begin{aligned}
g_{2}\left(y_{1}, y_{2} ; z_{1}, z_{2}\right)= & l_{p}^{2} \frac{1}{L^{2}} \sum_{i, j=1}^{N}\left[\alpha^{3}\left(\lambda_{i}^{F}-\lambda_{j}^{F}\right)^{2}+2\left|l_{p}\right| \alpha^{5} \xi_{p}\right. \\
& \left.\times\left(\lambda_{i}^{F}-\lambda_{j}^{F}\right)^{4}\right] X\left(\lambda_{i}^{F}, \lambda_{j}^{F}\right)
\end{aligned}
$$

where

$$
\begin{aligned}
X\left(\lambda_{i}^{F}, \lambda_{j}^{F}\right)= & \exp \left[-i \lambda_{i}^{F}\left(y_{1}^{F}-z_{1}^{F}\right)\right] \exp \left[-i \lambda_{j}^{F}\left(y_{2}^{F}-z_{2}^{F}\right)\right] \\
& +\exp \left[-i \lambda_{i}^{F}\left(y_{1}^{F}-z_{2}^{F}\right)\right] \\
& \times \exp \left[-i \lambda_{j}^{F}\left(y_{2}^{F}-z_{1}^{F}\right)\right] .
\end{aligned}
$$

We will use this expression to calculate the $p$-wave contacts.

\section{B. $p$-wave contacts}

In the thermodynamic limit, we may use the Fermi distribution function to evaluate the $p$-wave contacts through the relations given in (23)-(26). The modified Fermi distribution function with single-particle energy $\left(\lambda^{F} \alpha\right)^{2}$ is given by

$$
f\left(\lambda^{F}\right)=\frac{1}{1+\exp \left(\left[\left(\lambda^{F} \alpha\right)^{2}-A\right] / T\right)},
$$

where $T$ and $A$ are the temperature and the effective chemical potential of the 1D Fermi gas, respectively, and we have set the Boltzmann constant $k_{B}=1$. It satisfies the normalization 
condition $\int_{-\infty}^{\infty} f\left(\lambda^{F}\right) \frac{L}{2 \pi} d \lambda^{F}=N$, which includes the firstorder modification of $\left|l_{p}\right|$ and is equivalent to the corresponding result from thermodynamic Bethe ansatz equations (see Appendix C). Notice that the density of states, i.e., the number of states with fermionic momentum in the interval $\left(\lambda^{F}, \lambda^{F}+\right.$ $\left.d \lambda^{F}\right)$, is $(L / 2 \pi) f\left(\lambda^{F}\right) d \lambda^{F}$, such that the summation in the two-body correlation function (38) becomes an integral in the following form:

$$
\begin{aligned}
g_{2}\left(y_{1}, y_{2} ; z_{1}, z_{2}\right)= & \frac{l_{p}^{2}}{4 \pi^{2}} \int_{-\infty}^{\infty} d \lambda_{1}^{F} \int_{-\infty}^{\infty} d \lambda_{2}^{F} f\left(\lambda_{1}^{F}\right) f\left(\lambda_{2}^{F}\right) \\
& \times\left[\alpha^{3}\left(\lambda_{1}^{F}-\lambda_{2}^{F}\right)^{2}+2\left|l_{p}\right| \alpha^{5} \xi_{p}\right. \\
& \left.\times\left(\lambda_{1}^{F}-\lambda_{2}^{F}\right)^{4}\right] X\left(\lambda_{1}^{F}, \lambda_{2}^{F}\right) .
\end{aligned}
$$

For our convenience in calculation, we make a change of variable $\lambda_{1,2}^{F}=2 \pi n x_{1,2}$ and then obtain

$$
\begin{aligned}
g_{2}\left(y_{1}, y_{2} ; z_{1}, z_{2}\right)= & l_{p}^{2} 4 \pi^{2} n^{4} \int_{-\infty}^{\infty} d x_{1} \int_{-\infty}^{\infty} d x_{2} \mathcal{N}\left(x_{1}\right) \mathcal{N}\left(x_{2}\right) \\
& \times\left[\alpha^{3}\left(x_{1}-x_{2}\right)^{2}+2\left|l_{p}\right| \alpha^{5} \xi_{p}(2 \pi n)^{2}\right. \\
& \left.\times\left(x_{1}-x_{2}\right)^{4}\right] \times X\left(2 \pi n x_{1}, 2 \pi n x_{2}\right),
\end{aligned}
$$

where the function $\mathcal{N}(x)=f(2 \pi n x)$ is subject to the normalization condition $\int_{-\infty}^{\infty} \mathcal{N}(x) d x=1$. Substituting the above formula into Eqs. (23)-(26), we obtain explicitly the $p$-wave contacts in terms of the scattering length $\left|l_{p}\right|$ and the effective range $\xi_{p}$ :

$$
C_{2}=32 \pi N n^{3} l_{p}^{2}\left[\left(1-6\left|l_{p}\right| n\right) f_{2}+2\left|l_{p}\right| \xi_{p}(2 \pi n)^{2}\left(f_{4}+3 f_{2}^{2}\right)\right],
$$

$$
\begin{aligned}
C_{4}= & 32 \pi^{3} N n^{5} l_{p}^{2}\left[\left(1-10\left|l_{p}\right| n\right)\left(5 f_{4}+3 f_{2}^{2}\right)+2\left|l_{p}\right| \xi_{p}\right. \\
& \left.\times(2 \pi n)^{2}\left(5 f_{6}+27 f_{2} f_{4}\right)\right],
\end{aligned}
$$

with $f_{i}=\int_{-\infty}^{\infty} d x \mathcal{N}(x) x^{i}=\frac{1}{(2 \pi n)^{i+1}} \int_{0}^{\infty} d y \frac{y^{(i-1) / 2}}{1+e^{(y-A) / T}}$. We observe that in the weak-coupling regime the $p$-wave contacts $C_{2}$ and $C_{4}$ increase with both the scattering length $\left|l_{p}\right|$ and the effective range $\xi_{p}$. In addition, we have checked that indeed $C_{3}=0$ for the ground state and equilibrium states.

At low temperature $T \ll T_{d}$, here $T_{d}=\frac{\hbar^{2} n^{2}}{2 m k_{B}}$ is the degeneracy temperature, we can further calculate the contacts by the Sommerfeld expansion,

$$
\begin{aligned}
C_{2}= & \frac{8}{3} \pi N n^{3} l_{p}^{2}\left[\left(1-6\left|l_{p}\right| n\right)+\left(1+2\left|l_{p}\right| n\right) \frac{1}{4 \pi^{2}} \tau^{2}\right. \\
& \left.+\frac{16}{5}\left|l_{p}\right| \xi_{p}(\pi n)^{2}\left(1+\frac{5}{8 \pi^{2}} \tau^{2}\right)\right], \\
C_{4}= & \frac{8}{3} \pi^{3} N n^{5} l_{p}^{2}\left[\left(1-10\left|l_{p}\right| n\right)+\left(1-2\left|l_{p}\right| n\right) \frac{3}{4 \pi^{2}} \tau^{2}\right. \\
& \left.+\frac{132}{35}\left|l_{p}\right| \xi_{p}(\pi n)^{2}\left(1+\frac{14}{11 \pi^{2}} \tau^{2}\right)\right],
\end{aligned}
$$

with $\tau=T / T_{d}$ (see Appendix B). This indicates a simple relationship between the leading terms of $C_{2}$ and $C_{4}$, i.e., $C_{4}=C_{2}(n \pi)^{2}$. In this weak-coupling regime, the contact $C_{2}$ of the spinless $p$-wave Fermi gas behaves much like that of the
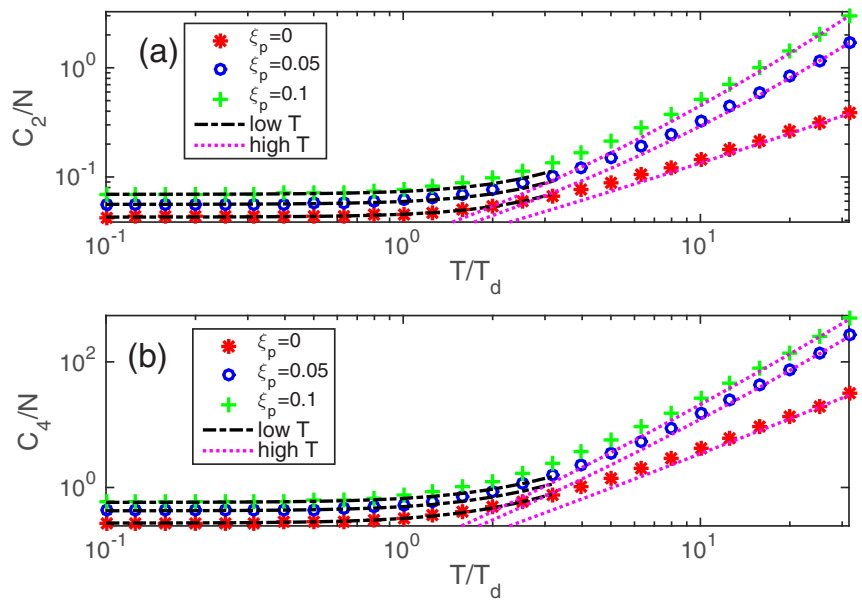

FIG. 2. The contacts for $p$-wave Fermi gas with weakly attractive interaction in the thermodynamic limit for $\left|l_{p}\right|=0.1$ and $n=1$. The marked lines are exact results (43) and (44). The black dash-dotted lines are for the low-temperature limit (45) and (46). The magenta dotted lines are for the high-temperature limit (47) and (48). The contacts $C_{2}$ and $C_{4}$ increase with both the temperature and the effective range.

Lieb-Liniger Bose gas with strong repulsion [48]. The contacts $C_{2}$ and $C_{4}$ increase smoothly as we increase the temperature. At relative high temperatures $T \gg T_{d}$, one can apply the Taylor series expansion with the distribution function $f_{i}$ under the condition $\exp (A / T) \ll 1$, and one obtains

$$
\begin{aligned}
C_{2}= & \frac{4}{\pi} \tau N n^{3} l_{p}^{2}\left[\left(1-2\left|l_{p}\right| n\right)+\left(1-4\left|l_{p}\right| n\right) \frac{\sqrt{2 \pi}}{2} \tau^{-1 / 2}\right. \\
& \left.+6\left|l_{p}\right| \xi_{p} n^{2} \tau\left(1+\frac{7 \sqrt{2 \pi}}{8} \tau^{-1 / 2}\right)\right], \\
C_{4}= & \frac{9}{\pi} \tau^{2} N n^{5} l_{p}^{2}\left[\left(1-2\left|l_{p}\right| n\right)+\left(1-4\left|l_{p}\right| n\right) \frac{19 \sqrt{2 \pi}}{24} \tau^{-1 / 2}\right. \\
& \left.+\frac{26}{3}\left|l_{p}\right| \xi_{p} n^{2} \tau\left(1+\frac{445 \sqrt{2 \pi}}{416} \tau^{-1 / 2}\right)\right] .
\end{aligned}
$$

In the above calculation, the weak interaction conditions require that $\left|l_{p}\right| / \Lambda \ll 1$, where the thermal de Broglie wavelength $\Lambda=\sqrt{\frac{4 \pi}{k_{B} T}}$. Namely, we request $T \ll T_{d} /\left(l_{p} n\right)^{2}$ and $\mathrm{C}_{2} /(\mathrm{Nn}) \ll 1$. We show the low- and high-temperature behavior of the contacts of the spinless Fermi gases with an attractive $p$-wave interaction in Fig. 2, where there is good agreement between the numerical results and the exact results obtained here.

\section{Energy derivatives with respect to the scattering length}

To relate the $p$-wave contact defined using asymptotic behavior of the momentum distribution to other physical observables, let us consider how $C_{2}$ is related to the derivative of energy with respect to $l_{p}$. Since the subleading $C_{4}$ term involves two contacts $C_{4}^{r}$ and $C_{4}^{c}$, and only the former is related to an energy derivative with respect to the effective range, we shall not discuss the corresponding adiabatic relation in this work. To that end, let us consider the $p$-wave pseudopotential 
of the following form $[49,50]$ :

$$
V(\vec{z})=-a_{p}^{1 D} \sum_{i<j}\left(\frac{\partial}{\partial z_{i}}-\frac{\partial}{\partial z_{j}}\right) \delta\left(z_{i}-z_{j}\right)\left(\frac{\partial}{\partial z_{i}}-\frac{\partial}{\partial z_{j}}\right) .
$$

According to the Hellman-Feynman theorem, the two-body correlation function is related to a derivative of energy with respect to $a_{p}^{1 \mathrm{D}}$, i.e., $g_{2}(0, \varepsilon ; 0, \varepsilon)=\frac{\left(a_{p}^{1 \mathrm{D}}\right)^{2}}{2 L} \frac{\partial E}{\partial a_{p}^{1 \mathrm{D}}}$. Combining the definition of contact $C_{2}$ with the effective scattering length $l_{p}=$ $a_{p}^{1 \mathrm{D}} / 2$, we may verify that the following relationship holds: $C_{2}=\frac{1}{\pi} \frac{\partial E}{\partial\left(-a_{p}^{1 D}\right)^{-1}}$. For a grand canonical ensemble, this relation can be rewritten as

$$
C_{2}=\left.\frac{L}{\pi} \frac{\partial P}{\partial\left(a_{p}^{1 D}\right)^{-1}}\right|_{\mu, T}
$$

where the pressure $P$ at finite temperatures and for general interaction strength can be obtained by thermodynamic Bethe ansatz (TBA); see Appendix C. By explicit calculation and comparison, one can show that Eq. (50) exactly reproduces the contact $C_{2}$ given in (45) and (47).

We first study the ground energy and its corresponding contact using the BA equations. When $\left|l_{p}\right| \ll L$ and $\xi_{p} \ll L$, the BA equations (8) for the ground state give a class of asymptotic solutions

$$
\lambda_{i}=\lambda_{i}^{F}\left\{1-2 n\left|l_{p}\right|+\left[4 n^{2}-2 \xi_{p}\left(n\left(\lambda_{i}^{F}\right)^{2}+3 E^{F} / L\right)\right] l_{p}^{2}\right\}
$$

up to the second order of $\left|l_{p}\right|$, where $\lambda_{i}^{F}$ and $E^{F}$ are quasimomentum and energy for 1D ideal Fermi gases, respectively. Consequently, we have the ground-state energy

$$
\begin{aligned}
E= & E^{F}\left\{1-4 n\left|l_{p}\right|\right. \\
& \left.+4\left[3 n^{2}-\xi_{p}\left(n \sum_{i=1}^{N}\left(\lambda_{i}^{F}\right)^{4} / E^{F}+3 E^{F} / L\right)\right] l_{p}^{2}\right\} .
\end{aligned}
$$

Taking derivative of the ground-state energy with respect to 1D scattering length, we obtain the contact

$$
\begin{aligned}
C_{2}= & \frac{1}{\pi} 8 n E^{F} l_{p}^{2}\left[1-6 n\left|l_{p}\right|\right. \\
& \left.\left.+2 \xi_{p}\left|l_{p}\right|\left(\sum_{i=1}^{N}\left(\lambda_{i}^{F}\right)^{4} / E^{F}+3 E^{F} / N\right)\right)\right] .
\end{aligned}
$$

In the thermodynamics limit, the contact $C_{2}=$ $\frac{8}{3} \pi n^{3} N l_{p}^{2}\left(1-6 n\left|l_{p}\right|+\frac{16}{5} \xi_{p}\left|l_{p}\right| \pi^{2} n^{2}\right)$ is consistent with expression given in (45) for the ground state.

In the following we consider the grand canonical ensemble in order to confirm the relation (50). For $l_{p}<0$, all solutions of the BA equations Eq. (7) are real. However, at finite temperatures, the eigenstates become degenerate. Following the approach introduced by Yang and Yang [51] in dealing with the thermodynamics of the 1D Bose gas, we determine the Gibbs free energy $G=E-T S-\mu N$ for the spinless Fermi gases with an attractive $p$-wave interaction by a minimization $G$ with respect to the BA root densities. In the Gibbs free energy, $S$ denotes entropy and $\mu$ is the chemical potential. It follows that

$$
\epsilon(\lambda)=\lambda^{2}-\mu-\frac{T}{2 \pi} \int_{-\infty}^{\infty} K(k-\lambda) \ln \{1+\exp [-\epsilon(k) / T]\} d k,
$$

where $\epsilon(\lambda)$ is the dressed energy characterizing the excitation energy. In the above equation, the kernel function $K(x)$ is given by

$$
K(x)=\frac{2\left|l_{p}\right|\left(\left|l_{p}\right| \xi_{p} x^{2}+1\right)}{\left(\left|l_{p}\right| \xi_{p} x^{2}-1\right)^{2}+l_{p}^{2} x^{2}} .
$$

The pressure $P$ can then be expressed as

$$
P=\frac{T}{2 \pi} \int_{-\infty}^{\infty} \ln \{1+\exp [-\epsilon(\lambda) / T]\} d \lambda .
$$

This gives the equation of states, from which the particle density and the compressibility are determined by $n=\frac{\partial P}{\partial \mu}$ and $\kappa=\frac{\partial n}{\partial \mu}$, respectively.

In the weak interaction limit $\left(\left|l_{p}\right| \ll 1\right)$, one can expand the kernel function $K(x)$ in powers of scattering length $\left|l_{p}\right|$. Up to the first few leading terms, the TBA equation (C1) can be simplified as

$$
\epsilon(\lambda)=B \lambda^{2}-A,
$$

where the effective chemical potential $A=\mu+2 Q_{0}\left|l_{p}\right|+$ $6 \xi_{p} Q_{2} l_{p}^{2}$ with the notations $B=1-6 \xi_{p} Q_{0} l_{p}^{2}$ and $Q_{j}=$ $\frac{T}{2 \pi} \int_{-\infty}^{\infty} k^{j} \ln \left\{1+\exp \left[-\frac{\epsilon(k)}{T}\right]\right\} d k$.

In the low-temperature limit $\left(T \ll T_{d}\right)$, the pressure $P$ up to the second order of $\left|l_{p}\right|$ reads

$$
\begin{aligned}
P= & \frac{2}{3 \pi} \mu^{3 / 2}\left\{1+2 \frac{\sqrt{\mu}}{\pi}\left|l_{p}\right|+\left(\frac{14}{3}+\frac{16 \pi}{5} \sqrt{\mu} \xi_{p}\right) \frac{\mu}{\pi^{2}} l_{p}^{2}\right. \\
& +\frac{\pi^{2}}{8} \frac{T^{2}}{\mu^{2}}\left[1+\frac{4}{3} \frac{\sqrt{\mu}}{\pi}\left|l_{p}\right|\right. \\
& \left.\left.+\left(\frac{10}{3}+\frac{48 \pi}{5} \sqrt{\mu} \xi_{p}\right) \frac{\mu}{\pi^{2}} l_{p}^{2}\right]\right\} .
\end{aligned}
$$

This gives an important indication of the deviation from the free fermions. From the formula (50), the contact is calculated in a straightforward way:

$$
\begin{aligned}
C_{2}= & \frac{8 L l_{p}^{2}}{3 \pi^{3}} \mu^{2}\left\{1+\left(\frac{14}{3}+\frac{16 \pi}{5} \sqrt{\mu} \xi_{p}\right) \frac{\sqrt{\mu}}{\pi}\left|l_{p}\right|+\frac{\pi^{2}}{12} \frac{T^{2}}{\mu^{2}}\right. \\
& \left.\times\left[1+\left(5+\frac{72 \pi}{5} \sqrt{\mu} \xi_{p}\right) \frac{\sqrt{\mu}}{\pi}\left|l_{p}\right|\right]\right\} .
\end{aligned}
$$

In this expression, the chemical potential can be expressed in terms of the particle density

$$
\begin{aligned}
\mu= & \pi^{2} n^{2}\left\{1-\frac{16}{3} n\left|l_{p}\right|+4 n^{2} l_{p}^{2}\left(5-\frac{16 \pi^{2}}{5} n \xi_{p}\right)\right. \\
& \left.+\frac{1}{12} \frac{T^{2}}{\pi^{2} n^{4}}\left[1-4 n^{2} l_{p}^{2}\left(1+8 \pi^{2} n \xi_{p}\right)\right]\right\} .
\end{aligned}
$$

The expression for $C_{2}$ in Eq. (59) is indeed consistent with expression (45), which was obtained from the many-body 
wave function. This confirms the universal relation (50). Furthermore, the compressibility has the analytical form

$$
\begin{aligned}
\kappa= & \frac{1}{2 n \pi^{2}}\left(1+\frac{1}{12 \pi^{2}} \frac{T^{2}}{n^{4}}\right)\left\{1+\left(8+\frac{2}{3 \pi^{2}} \frac{T^{2}}{n^{4}}\right) n\left|l_{p}\right|\right. \\
& \left.+\left[24+\frac{22}{3 \pi^{2}} \frac{T^{2}}{n^{4}}+\left(32 \pi^{2}+4 \frac{T^{2}}{n^{4}}\right) n \xi_{p}\right] n^{2} l_{p}^{2}\right\} .
\end{aligned}
$$

In the relative high-temperature regime, i.e., $T_{d} \ll T \ll$ $T_{d} /\left(l_{p} n\right)^{2}$, up to the second order of $\left|l_{p}\right|$, the pressure is given by

$$
\begin{aligned}
P= & \frac{1}{2} \frac{Z T^{3 / 2}}{\sqrt{\pi}}\left\{1-\frac{\sqrt{2}}{4} Z+\frac{Z \sqrt{T}}{\sqrt{\pi}}\left|l_{p}\right|\left(1-\frac{3 \sqrt{2}}{4} Z\right)\right. \\
& +\frac{3}{2 \pi} Z T l_{p}^{2}\left[Z-\frac{4 \sqrt{2}}{3} Z^{2}\right. \\
& \left.\left.+2 \sqrt{\pi T} \xi_{p}\left(1-\frac{9 \sqrt{2}}{16} Z\right)\right]\right\},
\end{aligned}
$$

where $Z=\exp (\mu / T)$ is the fugacity. From the relation (50), the contact is given explicitly

$$
\begin{aligned}
C_{2}= & \frac{T^{2} Z^{2} l_{p}^{2}}{2 \pi}\left\{1-\frac{3 \sqrt{2}}{4} Z+3 \sqrt{T / \pi}\left|l_{p}\right|\left[Z-\frac{4 \sqrt{2}}{3} Z^{2}\right.\right. \\
& \left.\left.+2 \sqrt{\pi T} \xi_{p}\left(1-\frac{9 \sqrt{2}}{16} Z\right)\right]\right\} .
\end{aligned}
$$

It is obvious that this result is consistent with expression (47) obtained from the many-body wave function (for details, see calculation in Appendix C). This alternative procedure provides a confirmation of the contact calculated by the manybody wave function in terms of the leading and subleading terms of the scattering length $\left|l_{p}\right|$, see Fig. 2 .

\section{Strong interaction limit}

The case $l_{p} \rightarrow-\infty$ is known as the fermionic TonksGirardeau gas $[52,53]$ at the $p$-wave resonance. In addition, if the effective range tends to zero, the wave function can be constructed from the noninteracting Bose gas with a sign function $[52,53]$. In the strong attraction regime, i.e., $\left|l_{p}\right| \gg$ $L+2 N \xi_{p}$, the quasimomentum distribution is similar to the 1D weakly interacting Bose gas, see review [15]. In this case, we assume that $\left|-\xi_{p} \lambda^{2}+1 /\right| l_{p}|| \ll|\lambda|$ and find that the phase shift $\theta(\lambda)$ in the BA equations (7) is close to $\pm \pi$, and thus the quasimomenta $\lambda_{i}$ 's are proportional to the square root of $1 /\left|l_{p}\right|$ in the form $\lambda_{i}=\beta_{i} H\left(\xi_{p}\right) / \sqrt{\left|l_{p}\right|}$ with $i=1, \ldots, N$. Here $\left\{\beta_{i}\right\}$ with $i=1,2, \ldots, N$ are determined by the sets of equations $\beta_{i}=\sum_{j=1(j \neq i)}^{N} 1 /\left(\beta_{i}-\beta_{j}\right)$, which are equivalent to the roots of the Hermite polynomial of degree $N$. $H\left(\xi_{p}\right)=\sqrt{2 /\left(L+2 N \xi_{p}\right)}$. The summation of all squares of $\beta_{i}$ gives $\sum_{i}^{N} \beta_{i}^{2}=N(N-1) / 2$, so that the energy of system is given by $E=N(N-1) /\left[\left(L+2 N \xi_{p}\right)\left|l_{p}\right|\right]$. It is obvious that the energy decreases with an increase of the scattering length $\left|l_{p}\right|$ and the effective range $\xi_{p}$.

The coefficients in the wave function can be rewritten as $a(P)=\exp \left(i B(P) / \sqrt{\left|l_{p}\right|}\right)$, with $B(P)=\sum_{i<j} F\left(\xi_{p}, \beta_{P_{i}}-\beta_{P_{j}}\right) \quad$ and $\quad F\left(\xi_{p}, \beta\right)=$ $\left[1-\xi_{p} H^{2}\left(\xi_{p}\right) \beta^{2}\right] /\left[H\left(\xi_{p}\right) \beta\right]$. The wave function in the domain $z_{1} \leqslant z_{2} \leqslant \cdots \leqslant z_{N}$ is reduced into the following form:

$$
\begin{aligned}
\psi(\vec{z})= & N !-\frac{1}{2} \sum_{P}\left[B(P)+H\left(\xi_{p}\right) \sum_{j=1}^{N} \beta_{P_{j}} z_{j}\right]^{2} \frac{1}{\left|l_{p}\right|} \\
& +O\left(\left|l_{p}\right|^{-3 / 2}\right)
\end{aligned}
$$

By calculating the quasilocal correlation function (see Appendix D), one obtains the leading terms for $C_{2}$ and $C_{4}$ as following

$$
C_{2}=\frac{2}{\pi} n(N-1), \quad C_{4}=\frac{n}{\left|l_{p}\right|} \frac{4(N-1)^{2}}{\pi L\left(1+2 n \xi_{p}\right)} .
$$

From the above expression, we see that the contact per particle $C_{2} / N$ is a finite value that depends only on the particle density in the thermodynamic limit. However, $C_{4}$ decreases with an increase of the scattering length $\left|l_{p}\right|$, and it goes to zero at the $p$-wave resonance $\left|l_{p}\right| \rightarrow \infty$. For $\xi_{p}=0, C_{2}$ is still given by (65), which can also be obtained from the energy derivative with respect to $\left|l_{p}\right|^{-1}[36,52,53]$.

\section{CONCLUSION}

In summary, using the exact many-body Bethe ansatz wave function, we obtained the high-momentum tail of a spinless Fermi gas interacting via $p$-wave scattering. We have found the leading and subleading coefficients of $1 / p^{2}$ and $1 / p^{4}$, i.e., the $p$-wave contacts, and give their analytic expressions in both weak and strong interaction limit. We also show by explicit calculation how the $p$-wave contacts are related to the shortdistance behavior of the two-body correlation functions. In addition, we have obtained the energetics and contacts in both the low- and high-temperature limits via thermodynamic Bethe ansatz.

\section{ACKNOWLEDGMENTS}

This work was supported by NSF of China under Grants No. 11704233, No. 11474189, and No. 11674201; and by Key NNSFC Grant No. 11534014 and the National Key R\&D Program of China No. 2017YFA0304500. S.Z. is supported by the Hong Kong Research Grants Council, Collaborative Research Fund (Grant No. C6026-16W) and GRF 17318316, and the Croucher Foundation under the Croucher Innovation Award. H.B.S. thanks Professor Vladimir Korepin for stimulating discussions and is grateful for support from the Society of Interdisciplinary Research (SOIREE).

\section{APPENDIX A: CORRELATION FUNCTION}

In the domain $z_{1} \leqslant z_{2} \leqslant \cdots \leqslant z_{N}$, the wave function has the following form:

$$
\psi(\vec{z})=\sum_{P} a(P) e^{i \sum_{j=1}^{N} \lambda_{P_{j}} z_{j}}
$$


For weak interaction, the coefficients $a(P)$ in above wave function can be expanded up to the second order of $\left|l_{p}\right|$ :

$$
\begin{aligned}
a(P) \approx & (-1)^{P} \prod_{i<j}\left\{1-\left|l_{p}\right| i\left(\lambda_{P_{i}}-\lambda_{P_{j}}\right)-\frac{1}{2} l_{p}^{2}\right. \\
& \left.\times\left(\lambda_{P_{i}}-\lambda_{P_{j}}\right)^{2}\left[1+2 i \xi_{p}\left(\lambda_{P_{i}}-\lambda_{P_{j}}\right)\right]\right\} .
\end{aligned}
$$

When $z_{2}=z_{1}+\varepsilon$, the coefficients $a\left(P_{1} P_{2} P_{3} \ldots P_{N}\right)$ and $a\left(P_{2} P_{1} P_{3} \ldots P_{N}\right)$ share the same plane wave $e^{i\left(\lambda_{P_{1}}+\lambda_{P_{2}}\right) z_{1}+i \sum_{j=3}^{N} \lambda_{P_{j}} z_{j}}$, so we calculate the summation of them

$$
\begin{aligned}
& a\left(P_{1} P_{2} P_{3} \ldots P_{N}\right)+a\left(P_{2} P_{1} P_{3} \ldots P_{N}\right) \\
& \approx 2(-1)^{P}\left(\frac{\left|l_{p}\right|}{i} \lambda_{P_{12}}+\frac{l_{p}^{2} \xi_{p}}{i} \lambda_{P_{12}}^{3}\right) \prod_{i<j=3}\left(1+\frac{\left|l_{p}\right|}{i} \lambda_{P_{i j}}\right) \\
& \approx 2(-1)^{P}\left(\frac{\left|l_{p}\right|}{i} \lambda_{P_{12}}+\frac{l_{p}^{2} \xi_{p}}{i} \lambda_{P_{12}}^{3}-l_{p}^{2} \lambda_{P_{12}} \sum_{i<j=3} \lambda_{P_{i j}}\right) \\
& =2(-1)^{P} \lambda_{P_{12}}\left\{\frac{\left|l_{p}\right|}{i}+l_{p}^{2}\left[\frac{\xi_{p}}{i} \lambda_{P_{12}}^{2}-(N-2)\left(\lambda_{P_{1}}+\lambda_{P_{2}}\right)\right.\right. \\
& \left.\left.-\sum_{i=3}^{N}(N+1-2 i) \lambda_{P_{i}}\right]\right\},
\end{aligned}
$$

in which $\quad \lambda_{P_{i j}}=\lambda_{P_{i}}-\lambda_{P_{j}} \quad$ and $\quad \sum_{i<j=3} \lambda_{P_{i j}}=$ $(N-2)\left(\lambda_{P_{1}}+\lambda_{P_{2}}\right)+\sum_{i=3}^{N}(N+1-2 i) \lambda_{P_{i}}$. And then the wave function for $z_{2}=z_{1}+\varepsilon$ is rewritten as

$$
\begin{aligned}
\psi(\vec{z}) \approx & \sum_{P}(-1)^{P} \lambda_{P_{12}}\left\{\frac{\left|l_{p}\right|}{i}+l_{p}^{2}\left[\frac{\xi_{p}}{i} \lambda_{P_{12}}^{2}-(N-2)\right.\right. \\
& \left.\left.\times\left(\lambda_{P_{1}}+\lambda_{P_{2}}\right)-\sum_{i=3}^{N}(N+1-2 i) \lambda_{P_{i}}\right]\right\} e^{i \sum_{j=1}^{N} \lambda_{P_{j}} z_{j}} \\
= & \psi^{(1)}(\vec{z})\left|l_{p}\right|+\psi^{(2)}(\vec{z}) l_{p}^{2},
\end{aligned}
$$

in which the wave function to the first order and the second order of $\left|l_{p}\right|$ are given by

$$
\begin{gathered}
\psi^{(1)}(\vec{z})=\sum_{P}(-1)^{P} \frac{\lambda_{P_{12}}}{i} e^{i \sum_{j=1}^{N} \lambda_{P_{j}} z_{j}} \\
=\sum_{P}(-1)^{P}\left(\partial_{z_{2}}-\partial_{z_{1}}\right) e^{i \sum_{j=1}^{N} \lambda_{P_{j}} z_{j}} \\
=\left(\partial_{z_{2}}-\partial_{z_{1}}\right) \psi^{(0)}(\vec{z}), \\
\psi^{(2)(\vec{z})=} \sum_{P}(-1)^{P} \lambda_{P_{12}}\left[\frac{\xi_{p}}{i} \lambda_{P_{12}}^{2}-(N-2)\left(\lambda_{P_{1}}+\lambda_{P_{2}}\right)\right. \\
\left.-\sum_{i=3}^{N}(N+1-2 i) \lambda_{P_{i}}\right] e^{i \sum_{j=1}^{N} \lambda_{P_{j}} z_{j}}
\end{gathered}
$$

$$
\begin{aligned}
= & \sum_{P} i(-1)^{P}\left(\partial_{z_{2}}-\partial_{z_{1}}\right)\left[i \xi_{p}\left(\partial_{z_{2}}-\partial_{z_{1}}\right)^{2}\right. \\
& \left.+i(N-2)\left(\partial_{z_{2}}+\partial_{z_{1}}\right)+i \sum_{i=3}^{N}(N+1-2 i) \partial_{z_{i}}\right] \\
& \times e^{i \sum_{j=1}^{N} \lambda_{P_{j}} z_{j}} \\
= & -\left[\xi_{p}\left(\partial_{z_{2}}-\partial_{z_{1}}\right)^{2}+(N-2)\left(\partial_{z_{2}}+\partial_{z_{1}}\right)\right. \\
& \left.+\sum_{i=3}^{N}(N+1-2 i) \partial_{z_{i}}\right] \psi^{(1)}(\vec{z}),
\end{aligned}
$$

with the zeroth-order wave function $\psi^{(0)}(\vec{z})=$ $\sum_{P}(-1)^{P} e^{i \sum_{j=1}^{N} \lambda_{P_{j}} z_{j}}$.

The correlation function without normalization for infinitesimal $y_{1}, y_{2} ; z_{1}, z_{2}$ is calculated by the above wave function,

$$
\begin{aligned}
& g_{2}\left(y_{1}, y_{2} ; z_{1}, z_{2}\right) \\
& =\int_{0}^{L} d z_{3} \cdots \int_{0}^{L} d z_{N} \psi^{*}\left(y_{1}, y_{2}, z_{3}, \ldots\right) \psi(\vec{z}) \\
& =(N-2) ! \int_{0 \leqslant z_{3} \leqslant \cdots \leqslant z_{N} \leqslant L} \psi^{*}\left(y_{1}, y_{2}, z_{3}, \ldots\right) \psi(\vec{z}) \\
& \approx(N-2) ! \rho^{(\Delta)} l_{p}^{2}-(N-2) !\left|l_{p}^{3}\right|\left\{\xi _ { p } \left[\left(\partial_{z_{2}}-\partial_{z_{1}}\right)^{2}\right.\right. \\
& \left.\left.\quad+\left(\partial_{y_{2}}-\partial_{y_{1}}\right)^{2}\right]+(N-2)\left(\partial_{z_{2}}+\partial_{z_{1}}+\partial_{y_{2}}+\partial_{y_{1}}\right)\right\} \rho^{(\Delta)} \\
& \quad-(N-2) !\left|l_{p}^{3}\right| \sum_{i=3}^{N}(N+1-2 i) \int_{0}^{L} \partial_{z_{i}} \rho_{i}^{(\Delta)}\left(z_{i}\right) d z_{i},
\end{aligned}
$$

in which

$$
\begin{gathered}
\rho^{(\Delta)} \equiv \int_{0 \leqslant z_{3} \leqslant \cdots \leqslant z_{N} \leqslant L} \psi^{(1) *}\left(y_{1}, y_{2}, z_{3}, \ldots\right) \psi^{(1)}(\vec{z}) d z_{3} \ldots d z_{N}, \\
\rho_{l}^{(\Delta)}\left(z_{l}\right) \equiv \int_{0 \leqslant z_{3} \leqslant \cdots \leqslant z_{N} \leqslant L} \psi^{(1) *}\left(y_{1}, y_{2}, z_{3}, \ldots\right) \psi^{(1)}(\vec{z}) \\
\quad \times d z_{3} \ldots d z_{l-1} d z_{l+1} \ldots d z_{N},
\end{gathered}
$$

with $3 \leqslant l \leqslant N$. For the first term in the correlation function (A7), we define the following derivatives:

$$
\begin{aligned}
g_{2}^{(2)} & \left(y_{1}, y_{2} ; z_{1}, z_{2}\right) \\
\equiv & (N-2) ! \rho^{(\Delta)} l_{p}^{2} \\
= & l_{p}^{2}\left(\partial_{y_{2}}-\partial_{y_{1}}\right)\left(\partial_{z_{2}}-\partial_{z_{1}}\right)(N-2) ! \\
& \times \int_{0 \leqslant z_{3} \leqslant \cdots \leqslant z_{N} \leqslant L} \psi^{(0) *}\left(y_{1}, y_{2}, z_{3}, \ldots\right) \psi^{(0)}(\vec{z}) d z_{3} \ldots d z_{N} \\
= & l_{p}^{2}\left(\partial_{y_{2}}-\partial_{y_{1}}\right)\left(\partial_{z_{2}}-\partial_{z_{1}}\right) g_{2}^{(0)}\left(y_{1}, y_{2} ; z_{1}, z_{2}\right)
\end{aligned}
$$

with

$$
\begin{aligned}
& g_{2}^{(0)}\left(y_{1}, y_{2} ; z_{1}, z_{2}\right) \\
& \quad=(N-2) \int_{0}^{L} d z_{3} \cdots \int_{0}^{L} d z_{N}\left[\psi^{(0)}\left(y_{1}, y_{2}, z_{3}, \ldots\right)\right]^{*} \psi^{(0)}(\vec{z}) .
\end{aligned}
$$


The last term in the correlation function (A7) can be proved to be zero with

$$
\int_{0}^{L} \partial_{z_{i}} \rho_{i}^{(\Delta)}\left(z_{i}\right) d z_{i}=\rho_{i}^{(\Delta)}(L)-\rho_{i}^{(\Delta)}(0)=0 .
$$

At last, one can obtain the correlation function

$$
\begin{aligned}
g_{2}\left(y_{1}, y_{2} ; z_{1}, z_{2}\right)= & \left\{1-\left|l_{p}\right| \xi_{p}\left[\left(\partial_{z_{2}}-\partial_{z_{1}}\right)^{2}+\left(\partial_{y_{2}}-\partial_{y_{1}}\right)^{2}\right]\right\} \\
& \times g_{2}^{(2)}\left(y_{1}, y_{2} ; z_{1}, z_{2}\right) .
\end{aligned}
$$

The Bethe ansatz equations (8) for weak attractive interaction $\left(\left|l_{p}\right| \ll L\right)$ exhibit the asymptotic solution $\lambda_{i}=\lambda_{i}^{F} \alpha$ for total momentum $\sum_{i=1}^{N} \lambda_{i}=0$, where $\lambda_{i}^{F}=2 \pi J_{i} / L, \alpha=1-$ $2\left|l_{p}\right| n$, and $J_{i}$ are (half-)integers satisfying $J_{1}<J_{2}<\cdots<$ $J_{N}$. Under the scaling $z_{i}=z_{i}^{F} / \alpha$, the zeroth-order correlation function is simplified to

$$
\begin{aligned}
g_{2}^{0}\left(y_{1}, y_{2} ; z_{1}, z_{2}\right) \approx & \alpha^{2-N} \int_{0}^{L} \psi^{F}\left(y_{1}^{F}, y_{2}^{F}, \ldots, z_{N}^{F}\right) \\
& \times \psi^{F}\left(\overrightarrow{z^{F}}\right) d z_{3}^{F} \cdots d z_{N}^{F},
\end{aligned}
$$

with the wave function of the ideal Fermi gas $\psi^{F}\left(\overrightarrow{z^{F}}\right)=$ $\sum_{Q}(-1)^{Q} e^{i \sum_{j=1}^{N} k_{Q_{j}}^{F} z_{j}^{F}}$. Meanwhile, using the normalization factor [48],

$$
\begin{aligned}
& \int_{0}^{L} d z_{1} \cdots \int_{0}^{L} d z_{N}|\Psi(\vec{z})|^{2} \\
& =\alpha^{1-N} \int_{0}^{L} d z_{1} \cdots \int_{0}^{L} d z_{N}\left|\psi^{(0)}(\vec{z})\right|^{2} \\
& \approx \alpha^{1-N} \int_{0}^{L} d z_{1}^{F} \cdots \int_{0}^{L} d z_{N}^{F}\left|\psi^{F}\left(\vec{z}^{F}\right)\right|^{2},
\end{aligned}
$$

the zeroth-order correlation function with normalization factor has the following form:

$$
\begin{aligned}
& \frac{g_{2}^{0}\left(y_{1}, y_{2} ; z_{1}, z_{2}\right)}{\alpha N(N-1)} \\
& =\frac{\int_{0}^{L} d z_{3}^{F} \cdots \int_{0}^{L} d z_{N}^{F} \psi^{F}\left(y_{1}^{F}, y_{2}^{F}, \ldots, z_{N}^{F}\right) \psi^{F}\left(\overrightarrow{z^{F}}\right)}{\int_{0}^{L} d z_{1}^{F} \cdots \int_{0}^{L} d z_{N}^{F}\left|\psi^{F}\left(\overrightarrow{z^{F}}\right)\right|^{2}} .
\end{aligned}
$$

Since $\psi^{F}\left(\overrightarrow{z^{F}}\right)$ is a Slater determinant, thus one can obtain the following result by Wick's theorem:

$$
\begin{aligned}
g_{2}^{0}\left(y_{1}, y_{2} ; z_{1}, z_{2}\right)= & \alpha\left[G\left(y_{1}^{F}, z_{1}^{F}\right) G\left(y_{2}^{F}, z_{2}^{F}\right)\right. \\
& \left.-G\left(y_{1}^{F}, z_{2}^{F}\right) G\left(y_{2}^{F}, z_{1}^{F}\right)\right],
\end{aligned}
$$

where the single-particle reduced density matrix of ideal fermions is given by $G(y, z)=\frac{1}{L} \sum_{i=1}^{N} \exp \left[-i \lambda_{i}^{F}(y-z)\right]$. By using the above result, the two-body correlation function has the following form:

$$
\begin{aligned}
& g_{2}^{(2)}\left(y_{1}, y_{2} ; z_{1}, z_{2}\right) \\
& =l_{p}^{2}\left(\partial_{y_{2}}-\partial_{y_{1}}\right)\left(\partial_{z_{2}}-\partial_{z_{1}}\right) g_{2}^{(0)}\left(y_{1}, y_{2} ; z_{1}, z_{2}\right) \\
& =l_{p}^{2} \alpha^{3}\left(\partial_{y_{2}^{F}}-\partial_{y_{1}^{F}}\right)\left(\partial_{z_{2}^{F}}-\partial_{z_{1}^{F}}\right)\left[G\left(y_{1}^{F}, z_{1}^{F}\right) G\left(y_{2}^{F}, z_{2}^{F}\right)\right. \\
& \left.\quad-G\left(y_{1}^{F}, z_{2}^{F}\right) G\left(y_{2}^{F}, z_{1}^{F}\right)\right]
\end{aligned}
$$

$$
=l_{p}^{2} \frac{1}{L^{2}} \sum_{i, j=1}^{N} \alpha^{3}\left(\lambda_{i}^{F}-\lambda_{j}^{F}\right)^{2} X\left(\lambda_{i}^{F}, \lambda_{j}^{F}\right),
$$

where

$$
\begin{aligned}
X\left(\lambda_{i}^{F}, \lambda_{j}^{F}\right)= & \exp \left[-i \lambda_{i}^{F}\left(y_{1}^{F}-z_{1}^{F}\right)\right] \exp \left[-i \lambda_{j}^{F}\left(y_{2}^{F}-z_{2}^{F}\right)\right] \\
& +\exp \left[-i \lambda_{i}^{F}\left(y_{1}^{F}-z_{2}^{F}\right)\right] \\
& \times \exp \left[-i \lambda_{j}^{F}\left(y_{2}^{F}-z_{1}^{F}\right)\right] .
\end{aligned}
$$

Finally, we have the two-body correlation function (38) in the main text, namely,

$$
\begin{aligned}
g_{2}\left(y_{1}, y_{2} ; z_{1}, z_{2}\right)= & l_{p}^{2} \frac{1}{L^{2}} \sum_{i, j=1}^{N}\left[\alpha^{3}\left(\lambda_{i}^{F}-\lambda_{j}^{F}\right)^{2}+2\left|l_{p}\right| \alpha^{5} \xi_{p}\right. \\
& \left.\times\left(\lambda_{i}^{F}-\lambda_{j}^{F}\right)^{4}\right] X\left(\lambda_{i}^{F}, \lambda_{j}^{F}\right) .
\end{aligned}
$$

\section{APPENDIX B: $p$-WAVE CONTACTS, $C_{2}$ AND $C_{4}$}

For the low temperature, $A / T \gg 1$, according to Sommerfeld expansion, the function $f_{i}=\int_{-\infty}^{\infty} d x \mathcal{N}(x) x^{i}=$ $\frac{1}{(2 \pi n \alpha)^{i+1}} \int_{0}^{\infty} d y \frac{y^{(i-1) / 2}}{1+e^{(y-A) / T}}$ is expanded with respect to the temperature

$$
\begin{aligned}
f_{i}= & \frac{1}{(2 \pi n \alpha)^{i+1}}\left[\frac{2}{i+1} A^{(i+1) / 2}\right. \\
& \left.+\frac{\pi^{2}(i-1)}{12} T^{2} A^{(i-3) / 2}+\cdots\right] .
\end{aligned}
$$

From the normalization condition $f_{0}=1$, the effective chemical potential $A$ can be solved by the iteration method

$$
A \approx(\pi n \alpha)^{2}\left(1+\frac{1}{12 \pi^{2}} \frac{\tau^{2}}{\alpha^{4}}\right)
$$

with $\tau=T / n^{2}$. And putting the effective chemical potential into $f_{i}, f_{i}$ is expressed with $\tau$ and $\alpha$,

$$
f_{i}=\frac{1}{(i+1) 2^{i}}\left[1+\frac{i(i+1)}{24 \pi^{2}} \frac{\tau^{2}}{\alpha^{4}}+\cdots\right],
$$

and then one can obtain the contact $C_{2}$ and $C_{4}$,

$$
\begin{aligned}
C_{2}= & \frac{8}{3}\left|l_{p}\right|^{2} \pi n^{3} N\left[\left(1-6\left|l_{p}\right| n\right)+\left(1+2\left|l_{p}\right| n\right) \frac{1}{4 \pi^{2}} \tau^{2}\right. \\
& \left.+\frac{16}{5}\left|l_{p}\right| \xi_{p}(\pi n)^{2}\left(1+\frac{5}{8 \pi^{2}} \tau^{2}\right)\right], \\
C_{4}= & \frac{8}{3}\left|l_{p}\right|^{2} \pi^{3} n^{5} N\left[\left(1-10\left|l_{p}\right| n\right)+\left(1-2\left|l_{p}\right| n\right) \frac{3}{4 \pi^{2}} \tau^{2}\right. \\
& \left.+\frac{132}{35}\left|l_{p}\right| \xi_{p}(\pi n)^{2}\left(1+\frac{14}{11 \pi^{2}} \tau^{2}\right)\right] .
\end{aligned}
$$

For the relative high temperature which requires effective fugacity $Z_{A} \equiv \exp (A / T) \ll 1$, the integral $f_{i}$ is expanded by effective fugacity:

$$
\begin{aligned}
f_{i} & \approx \frac{1}{(2 \pi n \alpha)^{i+1}} \int_{0}^{\infty} d y y^{(i-1) / 2} Z_{A} e^{-y / T}\left(1-Z_{A} e^{-y / T}\right) \\
& =\frac{T^{(i+1) / 2}}{(2 \pi n \alpha)^{i+1}} \Gamma\left(\frac{i+1}{2}\right) Z_{A}\left(1-2^{-(i+1) / 2} Z_{A}\right)
\end{aligned}
$$


From the normalization condition $f_{0}=1$, the effective fugacity can be solved by the iteration method,

$$
Z_{A} \approx \frac{2 \sqrt{\pi} \alpha}{\tau^{1 / 2}}\left(1+\frac{\sqrt{2 \pi} \alpha}{\tau^{1 / 2}}\right)
$$

and then one obtains the contact $C_{2}$ and $C_{4}$ :

$$
\begin{aligned}
C_{2}= & \frac{4}{\pi} l_{p}^{2} N n^{3} \tau\left[\left(1-2\left|l_{p}\right| n\right)+\left(1-4\left|l_{p}\right| n\right) \frac{\sqrt{2 \pi}}{2} \tau^{-1 / 2}\right. \\
& \left.+6\left|l_{p}\right| \xi_{p} T\left(1+\frac{7 \sqrt{2 \pi}}{8} \tau^{-1 / 2}\right)\right], \\
C_{4}= & \frac{9}{\pi} l_{p}^{2} N n^{3} \tau^{2}\left[\left(1-2\left|l_{p}\right| n\right)+\left(1-4\left|l_{p}\right| n\right) \frac{19 \sqrt{2 \pi}}{24} \tau^{-1 / 2}\right. \\
& \left.+\frac{26}{3}\left|l_{p}\right| \xi_{p} T\left(1+\frac{445 \sqrt{2 \pi}}{416} \tau^{-1 / 2}\right)\right] .
\end{aligned}
$$

\section{APPENDIX C: THE THERMODYNAMIC BETHE ANSATZ EQUATIONS}

The thermodynamic Bethe ansatz equation for $p$-wave fermions in one dimension can be written as

$$
\epsilon(\lambda)=\lambda^{2}-\mu-\frac{T}{2 \pi} \int_{-\infty}^{\infty} K(k-\lambda) \ln \left\{1+\exp \left[-\frac{\epsilon(k)}{T}\right]\right\} d k
$$

with $K(x)=\frac{2\left|l_{p}\right|\left(\left|l_{p}\right| \xi_{p} x^{2}+1\right)}{\left(\left|l_{p}\right| \xi_{p} x^{2}-1\right)^{2}+l_{p}^{2} x^{2}}$. The pressure is defined as

$$
P=\frac{T}{2 \pi} \int_{-\infty}^{\infty} \ln \{1+\exp [-\epsilon(\lambda) / T]\} d \lambda .
$$

The grand canonical potential, the particle density per length, and the contact are given by $\Omega=-P L, n=\frac{\partial P}{\partial \mu}$, and $C_{2}=$ $\frac{2 L}{\pi} l_{p}^{2} \frac{\partial P}{\partial\left|l_{p}\right|}$.

For weak interaction, $\left|l_{p}\right|<1 / n$, the kernel in the integral function of Eq. (C1) is expanded up to the second order of $\left|l_{p}\right|$,

$K(k-\lambda) \approx 2\left|l_{p}\right|+6 \xi_{p}\left|l_{p}\right|^{2} \lambda^{2}+12 \xi_{p}\left|l_{p}\right|^{2} \lambda k+6 \xi_{p}\left|l_{p}\right|^{2} k^{2}$,

and then the TBA equation (C1) is simplified as

$$
\epsilon(\lambda)=B \lambda^{2}-A,
$$

with the effective chemical potential $A=\mu+2\left|l_{p}\right| Q_{0}+$ $6 Q_{2} \xi_{p}\left|l_{p}\right|^{2}$ with the notations $B=1-6 Q_{0} \xi_{p}\left|l_{p}\right|^{2}$ and $Q_{j}=$ $\frac{T}{2 \pi} \int_{-\infty}^{\infty} k^{j} \ln \left\{1+\exp \left[-\frac{\epsilon(k)}{T}\right]\right\} d k$. Using the integral in part, $Q_{j}$ can be expressed by the polylogarithm

$$
Q_{j}=-\left(\frac{T}{B}\right)^{\frac{j+1}{2}} \frac{T}{\pi(j+1)} \Gamma\left(\frac{j+3}{2}\right) \operatorname{Li}_{\frac{j+3}{2}}\left(-\mathrm{e}^{A / T}\right),
$$

and using the properties of the polylogarithm $\mathrm{Li}_{i}\left(-\mathrm{e}^{A / T}\right)$, one obtains pressure, particle density, and contact with the following forms:

$$
P=-\frac{T^{3 / 2}}{2 \sqrt{\pi}} \operatorname{Li}_{\frac{3}{2}}\left(-\mathrm{e}^{\frac{A}{T}}\right)\left[1-\frac{3 T^{3 / 2}}{2 \sqrt{\pi}} \operatorname{Li}_{\frac{3}{2}}\left(-\mathrm{e}^{\frac{A}{T}}\right) \xi_{p}\left|l_{p}\right|^{2}\right]
$$

$$
\begin{gathered}
n=-\frac{T^{1 / 2}}{2 \sqrt{\pi}} \operatorname{Li}_{\frac{1}{2}}\left(-\mathrm{e}^{\frac{A}{T}}\right)\left(1+2\left|l_{p}\right| n\right) \\
C_{2}=\frac{2 L}{\pi} l_{p}^{2} n\left\{2 P-\left[\frac{3 T^{5 / 2}}{\sqrt{\pi}} \operatorname{Li}_{\frac{5}{2}}\left(-\mathrm{e}^{\frac{A}{T}}\right)-\frac{6 P^{2}}{n}\right] \xi_{p}\left|l_{p}\right|\right\},
\end{gathered}
$$

in which the expression of particle density is equivalent to previous normalization conditions of the modified Fermi-Dirac distribution function, and the contact $C_{2}$ is also consistent with the previous result from the many-body wave function.

In the low-temperature limit $\left(T \ll T_{d}\right)$, according to Sommerfeld expansion, $Q_{j}$ is expanded with respect to temperature:

$$
\begin{aligned}
Q_{j} \approx & \left(\frac{1}{B}\right)^{\frac{j+1}{2}} \frac{1}{\pi(j+1)}\left[\frac{2}{j+3} A^{(j+3) / 2}\right. \\
& \left.+\frac{j+1}{2} \frac{\pi^{2}}{6} T^{2} A^{(j-1) / 2}\right] .
\end{aligned}
$$

When the iteration method is applied, the pressure is rewritten as

$$
\begin{aligned}
P= & \frac{2}{3 \pi} \mu^{3 / 2}\left\{1+2 \frac{\sqrt{\mu}}{\pi}\left|l_{p}\right|+\left(\frac{14}{3}+\frac{16 \pi}{5} \sqrt{\mu} \xi_{p}\right) \frac{\mu}{\pi^{2}} l_{p}^{2}\right. \\
& \times \frac{\pi^{2}}{8} \frac{T^{2}}{\mu^{2}}\left[1+\frac{4}{3} \frac{\sqrt{\mu}}{\pi}\left|l_{p}\right|\right. \\
& \left.\left.+\left(\frac{10}{3}+\frac{48 \pi}{5} \sqrt{\mu} \xi_{p}\right) \frac{\mu}{\pi^{2}} l_{p}^{2}\right]\right\}
\end{aligned}
$$

According to formula $C_{2}=\frac{2 L}{\pi} l_{p}^{2} \frac{\partial P}{\partial\left|l_{p}\right|}$, the contact is

$$
\begin{aligned}
C_{2}= & \frac{8 L l_{p}^{2}}{3 \pi^{3}} \mu^{2}\left\{1+\left(\frac{14}{3}+\frac{16 \pi}{5} \sqrt{\mu} \xi_{p}\right) \frac{\sqrt{\mu}}{\pi}\left|l_{p}\right|+\frac{\pi^{2}}{12} \frac{T^{2}}{\mu^{2}}\right. \\
& \left.\times\left[1+\left(5+\frac{72 \pi}{5} \sqrt{\mu} \xi_{p}\right) \frac{\sqrt{\mu}}{\pi}\left|l_{p}\right|\right]\right\},
\end{aligned}
$$

in which chemical potential can be expressed by particle density with the form

$$
\mu=\pi^{2} n^{2}\left[1-\frac{16}{3} n\left|l_{p}\right|+\frac{1}{12} \frac{T^{2}}{\pi^{2} n^{4}}\right] .
$$

At last one obtains the contact

$$
\begin{aligned}
C_{2}= & \frac{8}{3} \pi l_{p}^{2} n^{3} N\left[\left(1-6\left|l_{p}\right| n\right)+\frac{1}{4} \frac{T^{2}}{\pi^{2} n^{4}}\left(1+2\left|l_{p}\right| n\right)\right. \\
& \left.+\frac{16}{5} n^{2} \pi^{2} \xi_{p}\left|l_{p}\right|\left(1+\frac{5}{8} \frac{T^{2}}{\pi^{2} n^{4}}\right)\right]
\end{aligned}
$$

This result is consistent with that from many-body wave function.

For the relative high temperature, the fugacity $Z=\mathrm{e}^{\mu / T} \ll 1$, $Q_{j}$ can be expanded by the fugacity

$$
\begin{aligned}
Q_{j}= & \left(\frac{T}{B}\right)^{\frac{j+1}{2}} \frac{T}{\pi(j+1)} \Gamma\left(\frac{j+3}{2}\right) \mathrm{e}^{A / T}\left[1-2^{-(j+3) / 2}\right. \\
& \left.\times \mathrm{e}^{A / T}+\mathrm{e}^{2 A / T} 3^{-(j+3) / 2}-\mathrm{e}^{3 A / T} 4^{-(j+3) / 2}\right] . \quad(\mathrm{C} 14)
\end{aligned}
$$


When the iteration method is applied, the pressure is rewritten as

$$
\begin{aligned}
P= & \frac{1}{2} \frac{Z T^{3 / 2}}{\sqrt{\pi}}\left[1-\frac{\sqrt{2}}{4} Z+\frac{Z \sqrt{T}}{\sqrt{\pi}}\left|l_{p}\right|\left(1-\frac{3 \sqrt{2}}{4} Z\right)+\frac{3}{2} Z^{2}\right. \\
& \left.\times \frac{T}{\pi} l_{p}^{2}\left(1-\frac{4 \sqrt{2}}{3} Z\right)+\frac{3 Z T^{3 / 2}}{\sqrt{\pi}} l_{p}^{2} \xi_{p}\left(1-\frac{9 \sqrt{2}}{16} Z\right)\right] .
\end{aligned}
$$

According to the formula $C_{2}=\frac{2 L}{\pi} l_{p}^{2} \frac{\partial P}{\partial\left|l_{p}\right|}$, the contact is

$$
\begin{aligned}
C_{2}= & \frac{L l_{p}^{2} Z^{2} T^{2}}{\pi^{2}}\left[1-\frac{3}{4} \sqrt{2} Z+\left(1-\frac{4 \sqrt{2}}{3} Z\right) \frac{3 Z \sqrt{T}}{\sqrt{\pi}}\left|l_{p}\right|\right. \\
& \left.+6\left|l_{p}\right| \xi_{p} T\left(1-\frac{9}{16} \sqrt{2} Z\right)\right],
\end{aligned}
$$

in which fugacity can be expressed in particle density

$$
Z=\frac{2 \sqrt{\pi} n}{\sqrt{T}}\left(1+\frac{\sqrt{2 \pi} n}{\sqrt{T}}\right)-\left(1+\frac{7 \sqrt{2 \pi} n}{4 \sqrt{T}}\right) \frac{8 \sqrt{\pi} n}{\sqrt{T}}\left|l_{p}\right| n .
$$

At last, one can obtain the contact

$$
\begin{aligned}
C_{2}= & \frac{4 l_{p}^{2} T n N}{\pi}\left[1-2 n\left|l_{p}\right|+\left(1-4 n\left|l_{p}\right|\right) \frac{\sqrt{2 \pi}}{2} \frac{n}{\sqrt{T}}\right. \\
& \left.+6\left|l_{p}\right| \xi_{p} T\left(1+\frac{7 \sqrt{2 \pi}}{8} \frac{n}{\sqrt{T}}\right)\right],
\end{aligned}
$$

which is also consistent with that from the many-body wave function.

\section{APPENDIX D: STRONG-COUPLING LIMIT}

The energy of system composed of $N$ spinless fermions can be expressed as $E=\sum_{i}^{N} \lambda_{i}^{2}$, and the quasimomentum $\lambda_{i}$ satisfies the BA equation for real $\lambda_{i}$,

$$
\lambda_{i} L=2 \pi n_{i}-\sum_{j=1}^{N} \theta\left(\lambda_{i}-\lambda_{j}\right) .
$$

Here the phase shift $\theta(\lambda)$ is a monotonic antisymmetric function defined by

$$
\theta(\lambda)=2 \arg \left(i \lambda-\xi_{p} \lambda^{2}+1 /\left|l_{p}\right|\right)
$$

and $n_{i}=i-(N+1) / 2$ for the ground state. In the strong attractive limit $\left|l_{p}\right| \gg 1 / L$, and we assume that $\mid-\xi_{p} \lambda^{2}+$ $1 /\left|l_{p}\right||\ll| \lambda \mid$, and then phase shift approaches $\pm \pi$ with $\theta(\lambda) \approx \pi \lambda /|\lambda|-2\left(\frac{x}{\lambda}-\xi_{p} \lambda\right)$ and $x \equiv 1 /\left|l_{p}\right|$. And then the BA equation becomes the new form,

$$
\lambda_{i} L=\sum_{j=1(j \neq i)}^{N}\left[\frac{2 x}{\lambda_{i}-\lambda_{j}}-2 \xi_{p}\left(\lambda_{i}-\lambda_{j}\right)\right] .
$$

For the ground state, the total momentum is zero, which means $\sum_{j} \lambda_{j}=0$, so the BA equation can be simplified as the new form

$$
\left(L+2 N \xi_{p}\right) \lambda_{i}=\sum_{j=1(j \neq i)}^{N} \frac{2 x}{\lambda_{i}-\lambda_{j}} .
$$

The solution of the above equation has the following form: $\lambda_{i}=\sqrt{\frac{2 x}{L+2 N \xi_{p}}} \beta_{i}$, with constant $\beta_{i}$ which is determined by a set of equations $\beta_{i}=\sum_{j=1(j \neq i)}^{N} \frac{1}{\beta_{i}-\beta_{j}}$. The previous assumption $\left|-\xi_{p} \lambda^{2}+1 /\right| l_{p}|| \ll|\lambda|$ requires that $\left|l_{p}\right| \gg L+2 N \xi_{p}$, which is the strong-coupling condition for the above solution. With the above solution, the energy of the system is

$$
E=\frac{2 /\left|l_{p}\right|}{L+2 N \xi_{p}} \sum_{i} \beta_{i}^{2}=\frac{N(N-1) /\left|l_{p}\right|}{L+2 N \xi_{p}}
$$

The coefficients in the wave function can be written as

$$
\begin{aligned}
a(P) & =(-1)^{P} \prod_{i<j}\left\{\exp \left[-i \theta\left(\lambda_{P_{i}}-\lambda_{P_{j}}\right)\right]\right\}^{1 / 2} \\
& =\exp \left[i \frac{\pi}{2}\left(1-(-1)^{P}\right)-\frac{i}{2} \sum_{i<j} \theta\left(\lambda_{P_{i}}-\lambda_{P_{j}}\right)\right] .
\end{aligned}
$$

In the strong attractive interaction limit, $\lambda_{i}=\frac{H\left(\xi_{p}\right)}{\sqrt{\left|l_{p}\right|}} \beta_{i}$ with $H\left(\xi_{p}\right)=\sqrt{\frac{2}{L+2 N \xi_{p}}}$, and the phase shift $\theta(\lambda) \approx \frac{\lambda}{|\lambda|} \pi-$ $2 F\left(\xi_{p}, \beta\right) \frac{1}{\sqrt{\left|l_{p}\right|}}$, with $F\left(\xi_{p}, \beta\right)=\frac{1}{\sqrt{\frac{2}{L+2 N \xi_{p}}} \beta}\left(1-\frac{2 \xi_{p}}{L+2 N \xi_{p}} \beta^{2}\right)$, and $F\left(\xi_{p},-\beta\right)=-F\left(\xi_{p}, \beta\right)$. The coefficient has the following form:

$$
a(P)=\exp \left[\frac{i}{\sqrt{\left|l_{p}\right|}} B(P)\right]
$$

with $B(P)=\sum_{i<j} F\left(\xi_{p}, \beta_{P_{i}}-\beta_{P_{j}}\right)$. The wave function in the domain $z_{1} \leqslant z_{2} \leqslant \cdots \leqslant z_{N}$ can be simplified as

$$
\begin{aligned}
& \psi(\vec{z}) \\
& =\sum_{P} \exp \left[\frac{i}{\sqrt{\left|l_{p}\right|}} B(P)\right] \exp \left[\frac{i}{\sqrt{\left|l_{p}\right|}} H\left(\xi_{p}\right) \sum_{j=1}^{N} \beta_{P_{j}} z_{j}\right] \\
& \approx N !-\frac{Y\left(\vec{z}, \xi_{p}\right)}{\left|l_{p}\right|}
\end{aligned}
$$

with $Y\left(\vec{z}, \xi_{p}\right)=\frac{1}{2} \sum_{P}\left[B(P)+H\left(\xi_{p}\right) \sum_{j=1}^{N} \beta_{P_{j}} z_{j}\right]^{2}$. The correlation function can be written as

$$
\begin{aligned}
& g_{2}\left(y_{1}, y_{2} ; z_{1}, z_{2}\right) \\
& =\frac{N(N-1) \int_{0}^{L} d z_{3} \cdots \int_{0}^{L} d z_{N} \psi^{*}\left(y_{1}, y_{2}, z_{3}, \ldots\right) \psi(\vec{z})}{\int_{0}^{L} d z_{1} \cdots \int_{0}^{L} d z_{N}|\Psi(\vec{z})|^{2}} \\
& \approx \\
& \quad \times\left[N !-\frac{Y(N-1)[(N-2) !] N !}{(N !)^{2} L^{N}} \int_{0 \leqslant z_{3} \leqslant \cdots \leqslant z_{N} \leqslant L} d z_{3} \cdots d z_{N}\right. \\
& \left|l_{p}\right|
\end{aligned}
$$


in which we suppose that $y_{1}, y_{2}, z_{1}, z_{2}$ is close to zero. The leading terms of contacts $C_{2}, C_{4}^{r}, C_{4}^{c}$, and $C_{4}$ have the following results:

$$
\begin{aligned}
& C_{2}=\frac{2 L}{\pi} g_{2}(0, \varepsilon ; 0, \varepsilon) \\
& \approx \frac{2 L}{\pi} \frac{N(N-1)(N !)^{2} L^{N-2}}{(N !)^{2} L^{N}} \\
&=\frac{2}{\pi} \frac{N(N-1)}{L}, \\
& C_{4}^{r}=-\frac{L}{\pi}\left[\operatorname{Re}\left(\partial_{z_{2}}-\partial_{z_{1}}\right)^{2} g_{2}\left(y_{2}-\varepsilon, y_{2} ; z_{1}, z_{2}\right)\right]_{y_{2}=z_{2}=z_{1}+\varepsilon}
\end{aligned}
$$

$$
\begin{aligned}
\approx & -\frac{L}{\pi} \frac{\frac{-1}{\left|l_{p}\right|}(N !)^{2} L^{N-2} \frac{2 N}{L+2 N \xi_{p}} N(N-1)}{(N !)^{2} L^{N}} \\
= & \frac{1}{\pi} \frac{1}{\left|l_{p}\right|} \frac{2 n N(N-1)}{L+2 N \xi_{p}}, \\
C_{4}^{c}= & \frac{L}{2 \pi}\left[\left(\partial_{y_{2}} \partial_{z_{2}}-2 \operatorname{Re} \partial_{z_{2}}^{2}\right) g_{2}\left(y_{2}-\varepsilon, y_{2} ; z_{2}-\varepsilon, z_{2}\right)\right]_{y_{2}=z_{2}} \\
\approx & \frac{1}{\left|l_{p}\right|} \frac{2 N(N-1)(N-2)}{\pi L\left(L+2 N \xi_{p}\right)}, \\
& C_{4}=C_{4}^{c}+C_{4}^{r}=\frac{1}{\left|l_{p}\right|} \frac{4 N(N-1)^{2}}{\pi L\left(L+2 N \xi_{p}\right)} .
\end{aligned}
$$

[21] F. Deuretzbacher, D. Becker, J. Bjerlin, S. M. Reimann, and L. Santos, Phys. Rev. A 90, 013611 (2014).

[22] S. Murmann, F. Deuretzbacher, G. Zürn, J. Bjerlin, S. M. Reimann, L. Santos, T. Lompe, and S. Jochim, Phys. Rev. Lett. 115, 215301 (2015).

[23] J. Levinsen, P. Massignan, G. M. Bruun, and M. M. Parish, Sci. Adv. 1, e1500197 (2015).

[24] L. Yang, L. Guan, and H. Pu, Phys. Rev. A 91, 043634 (2015).

[25] H. Hu, L. Pan, and S. Chen, Phys. Rev. A 93, 033636 (2016).

[26] Y. Liu, S. Chen, and Y. Zhang, Phys. Rev. A 95, 043628 (2017).

[27] L. Pan, Y. Liu, H. Hu, Y. Zhang, and S. Chen, Phys. Rev. B 96, 075149 (2017).

[28] S. Tan, Ann. Phys. 323, 2952 (2008); 323, 2971 (2008); 323, 2987 (2008).

[29] E. Braaten and L. Platter, Phys. Rev. Lett. 100, 205301 (2008).

[30] S. Zhang and A. J. Leggett, Phys. Rev. A 79, 023601 (2009).

[31] F. Werner, L. Tarruell, and Y. Castin, Eur. Phys. J. B 68, 401 (2009).

[32] E. Braaten and L. Platter, Laser Phys. 19, 550 (2009).

[33] J. T. Stewart, J. P. Gaebler, T. E. Drake, and D. S. Jin, Phys. Rev. Lett. 104, 235301 (2010).

[34] S. M. Yoshida and M. Ueda, Phys. Rev. Lett. 115, 135303 (2015).

[35] Z. Yu, J. H. Thywissen, and S. Zhang, Phys. Rev. Lett. 115, 135304 (2015).

[36] Y. Sekino, S. Tan, and Y. Nishida, Phys. Rev. A 97, 013621 (2018).

[37] X. Cui, Phys. Rev. A 94, 043636 (2016).

[38] X. Cui and H. Dong, Phys. Rev. A 94, 063650 (2016).

[39] S.-G. Peng, X.-J. Liu, and H. Hu, Phys. Rev. A 94, 063651 (2016).

[40] M. He, S. Zhang, H. M. Chan, and Q. Zhou, Phys. Rev. Lett. 116, 045301 (2016).

[41] C. Luciuk, S. Trotzky, S. Smale, Z. Yu, S. Zhang, and J. H. Thywissen, Nat. Phys. 12, 599 (2016).

[42] L. Pricoupenko, Phys. Rev. Lett. 100, 170404 (2008).

[43] A. Imambekov, A. A. Lukyanov, L. I. Glazman, and V. Gritsev, Phys. Rev. Lett. 104, 040402 (2010).

[44] Y. Hao, Y. Zhang, and S. Chen, Phys. Rev. A 76, 063601 (2007).

[45] L. Pan, S. Chen, and X. Cui, Phys. Rev. A 98, 011603(R) (2018).

[46] X.-L. Chen, X.-J. Liu, and H. Hu, Phys. Rev. A 94, 033630 (2016).

[47] M. Olshanii and V. Dunjko, Phys. Rev. Lett. 91, 090401 (2003). 
[48] E. Nandani, R. A. Römer, S. Tan, and X. W. Guan, New J. Phys. 18, 055014 (2016).

[49] M. Girardeau, H. Nguyen, and M. Olshanii, Opt. Commun. 243, 3 (2004).

[50] H. Grosse, E. Langmann, and C. Paufler, J. Phys. A: Math. Gen. 37, 4579 (2004).
[51] C. N. Yang and C. P. Yang, J. Math. Phys. 10, 1115 (1969).

[52] S. A. Bender, K. D. Erker, and B. E. Granger, Phys. Rev. Lett. 95, 230404 (2005).

[53] M. D. Girardeau and A. Minguzzi, Phys. Rev. Lett. 96, 080404 (2006). 